\title{
Toward the First Nonpeptidic Molecular Tong Inhibitor of Wild-Type and Mutated HIV-1 Protease Dimerization
}

\author{
Anamaria Vidu ${ }^{[a, c]}$ Laure Dufau $^{[b]}{ }^{[b u d o v i c ~ B a n n w a r t h}{ }^{[b]}$ Jean-Louis Soulier, ${ }^{[a]}$ Sames Sicsic, ${ }^{[a]}$ \\ Umberto Piarulli, ${ }^{[c]}$ Michèle Reboud-Ravaux, ${ }^{*[b]}$ and Sandrine Ongeri*a]
}

\begin{abstract}
Herein we describe the synthesis and HIV-1 protease (PR) in hibitory activity of 16 new peptidomimetic molecular tongs with a naphthalene scaffold. Their peptidic character was progressively decreased. Two of these molecules exhibited the best dimerization inhibition activity toward HIV-1 wild-type and multimutated ANAM-11 proteases obtained to date for
\end{abstract}

this class of molecules ( $40 \mathrm{~nm}$ for wild-type PR and $100 \mathrm{~nm}$ for ANAM-11 PR). Although the peptidic character of one molecular tong was completely suppressed, the mechanism of inhibition and inhibitory potency toward both proteases were maintained.

\section{Introduction}

Human immunodeficiency virus type 1 (HIV-1) protease (HIV$\mathrm{PR}$ ) is the enzyme responsible for post-translational processing of viral polyproteins and subsequent generation of the structural and functional proteins essential for viral replication. ${ }^{[1]}$ As such, HIV-PR is a major target for antiretroviral therapies. HIV$\mathrm{PR}$ is a homodimeric aspartyl protease, with two monomers of 99 residues each. The enzyme active site is located at the bottom of a cavity within the dimer interface. Access to this active site is monitored by the $\beta$ hairpin flaps. HIV-PR is active only in its dimeric form; because each monomer contributes one of the two catalytic aspartic residues (Asp25), dissociation of the enzyme homodimer results in a complete loss of catalytic activity. The protease homodimer is mainly stabilized by a four-stranded antiparallel $\beta$ sheet involving both the $\mathrm{N}$ - and $\mathrm{C}$ termini of each monomer (H-Pro1-Gln2-Ile3-Thr4 and Cys95Thr96-Leu97-Asn98-Phe99-OH). This region appears to be highly conserved in HIV-1 isolates. ${ }^{[2]}$ More than $50 \%$ of the hydrogen bonds along the dimer interface involve terminal residues $1-4$ and $96-99$ at the antiparallel $\beta$ sheet. ${ }^{[3]}$ The contacts in this region contribute to $75 \%$ of the total Gibbs free energy of HIV-PR dimerization. ${ }^{[4]}$ Many HIV-PR inhibitors (PIs) have been developed which target the active site. However, several mutations located within or outside of the HIV-PR active site result in lower affinity towards inhibitors, ${ }^{[5,6]}$ and alternative strategies to circumvent the cross-resistance of HIV-PR inhibitors are crucially needed. Recently approved PIs, tipranavir and darunavir, are active against HIV-1 protease variants resistant to multiple PIs. Data obtained using a FRET-based HIV-1 expression assay suggested that these molecules act as conventional protease inhibitors. They also block protease dimerization in cells, but do not dissociate already dimerized cellular proteases. $^{[7]}$ The protease termini $\beta$ sheet interface has been explored as a dimerization inhibition target, and known inhibitors that target this region are able to block or disrupt the formation of the homodimer. ${ }^{[8]} \mathrm{C}$ - and $\mathrm{N}$-terminal mimetic peptides ${ }^{[9-11]}$ and lipopeptides ${ }^{[12-14]}$ have proven to be efficient PR dimerization inhibitors. A bicyclic guanidinium group has been introduced between the peptidic and lipophilic moieties, ${ }^{[15]}$ and the interface peptides have also been cross-linked with flexible, ${ }^{[16]}$ semi-rigid ${ }^{[17]}$ or rigid spacers. ${ }^{[18-20]}$

Our strategy involved the synthesis of conformationally constrained, scaffold-based molecular tongs, attached to two peptidic strands by carboxypropyl linkers (Figure 1). The two peptidic strands were able to be suitably oriented by the scaffold, allowing putative formation of an antiparallel $\beta$ sheet with the C-terminal end of one HIV-1 PR monomer and resulting in an entropic benefit (Figure 1). ${ }^{[18]}$ Naphthalene or quinoline scaffolds with symmetrical or asymmetrical peptidic strands were efficient antidimers ( $K_{\text {id }}$ up to $80 \mathrm{~nm}$ ). ${ }^{[18,19]}$ The introduction of amino acid mimetic fragments, specifically a 5-amino-2-methoxybenzamide or a 3-amino-6-methylpyridin $(1 H)$-one group in a single strand, increased the metabolic stability of the molecular tongs without compromising their ability to inhibit wild-type and mutated HIV-1 proteases in vitro. ${ }^{[20]}$ These groups were selected to replace two amino acids, as they were hypothesized to provide the same array of hydrogen bonding groups as one edge of a peptidic $\beta$ strand. Our previous results

[a] Dr. A. Vidu, Dr. J.-L. Soulier, Prof. S. Sicsic, Dr. S. Ongeri Molécules Fluorées et Chimie Médicinale, BioCIS UMR-CNRS 8076, IPSIT Université Paris-Sud 11, Faculté de Pharmacie

5 rue Jean-Baptiste Clément, 92296, Châtenay-Malabry Cedex (France) Fax: $(+33) 146835740$

E-mail:Sandrine.Ongeri@u-psud.fr

[b] L. Dufau, Dr. L. Bannwarth, Prof. M. Reboud-Ravaux UPMC-UR4, Enzymologie Moléculaire et Fonctionnelle 7 Quai St Bernard 75252 Paris Cedex 05 (France)

Fax: $(+33)$ 144-275-140

E-mail:michele.reboud@upmc.fr

[c] Dr. A. Vidu, Prof. U. Piarulli

Università degli Studi dell'Insubria, Dipartimento di Scienze Chimiche e Ambientali, Via Valleggio, 11, 22100 Como (Italy)

Supporting information for this article is available on the WWW under http://dx.doi.org/10.1002/cmdc.201000308. 


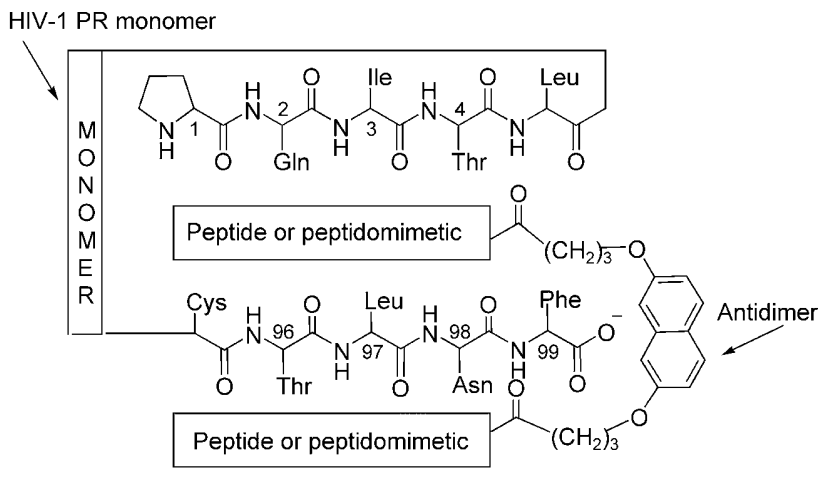

Figure 1. Schematic representation of a putative HIV-1 monomer-molecular tong complex.
We also report the first nonpeptidic molecular tong 15 that acts as an antidimer.

\section{Results and Discussion}

\section{Chemistry}

Protected peptidomimetic strand $\mathbf{1 8}$ was obtained by coupling 2-(3-amino-2-oxopyridin-1-(2H)-yl)acetamide $17^{[20]}$ to $\mathrm{N}$-Boc- $\mathrm{N}$ Z-Lys-OH, using HBTU and HOBt as coupling agents, in good yield (Scheme 1). Compound $\mathbf{1 8}$ was selectively deprotected at the $\alpha$-nitrogen by acidic hydrolysis (TFA in $\mathrm{CH}_{2} \mathrm{Cl}_{2}$ ) to afford 19, or at the $\varepsilon$-nitrogen by hydrogenolysis to give 20 , both in excellent yields (Scheme 1). suggest that the flexibility of valine is required for formation of the hydrogen bonds in a fourstranded antiparallel $\beta$ sheet, which involves both the $\mathrm{N}$ - and C-termini of the PR monomer. ${ }^{[20]}$

This report describes the synthesis and enzyme inhibitory activity against wild-type and mutated HIV-1 proteases of new molecular tongs with amino acid mimetic fragments in one (molecules 1-6, Figure 2) or two (molecules 7-16, Figure 2) strands. We decreased the peptidic character of the molecular tongs by introducing peptidomimetic fragments, first replacing two and then three amino acids in one or both strands. The 5amino-2-methoxybenzamide or 3-amino-6-methylpyridin(1H)-one groups were first attached to the carboxypropyl linker through a valine or lysine residue for molecules 1-2 and 7-12. In order to totally suppress the peptidic character of the strands, and to increase flexibility between the scaffold and the mimetic unit, the amino acid residue (valine or lysine) was replaced by a longer and more flexible moiety. The mimetic unit was attached to the carboxypropyl linker through the $\varepsilon$-amino group of a lysine residue (molecules 3-6 and 1316). We describe herein the most potent antidimeric molecular tongs $\mathbf{1}$ and $\mathbf{2}$ obtained to date for this class of molecules.
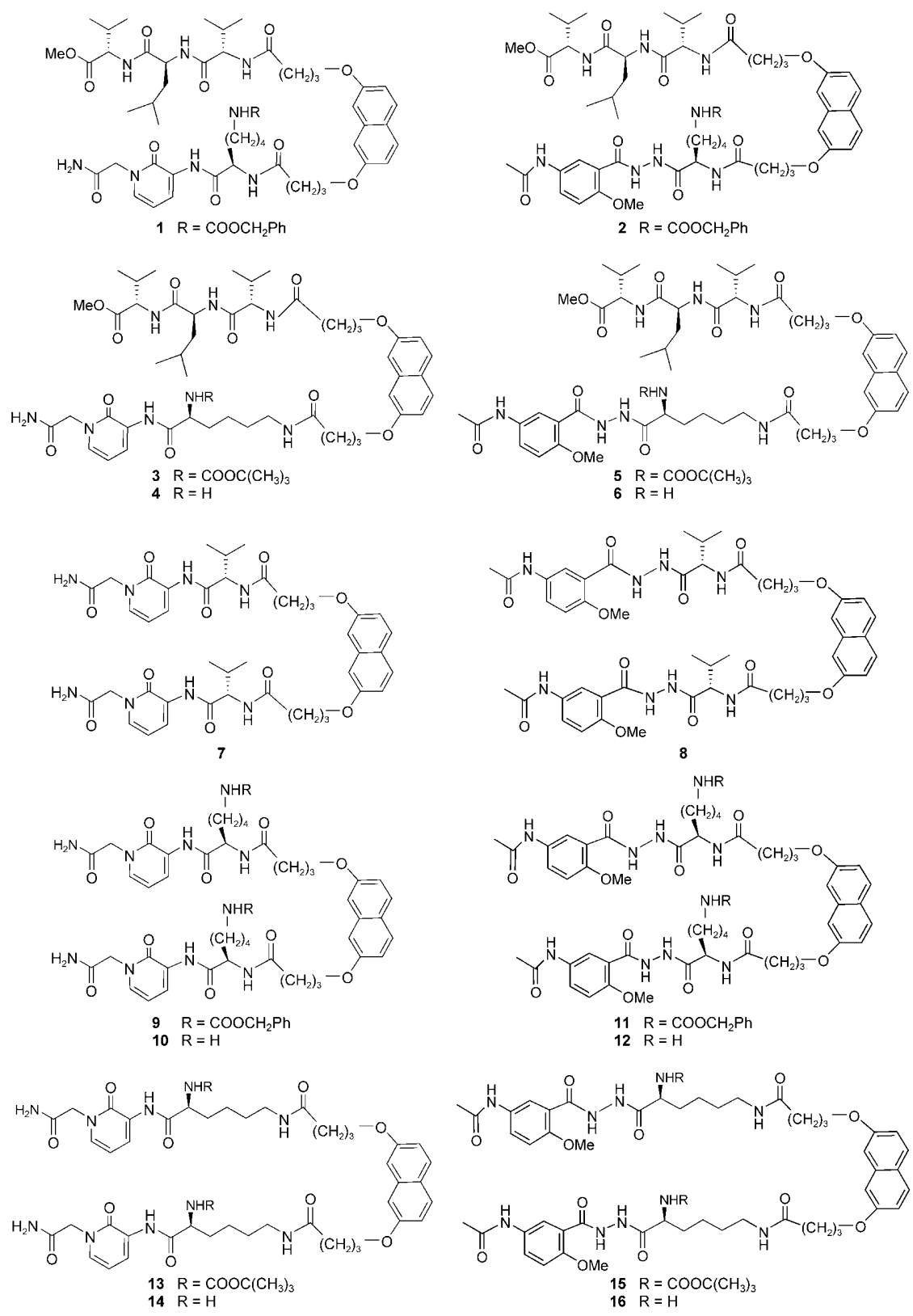

Figure 2. Molecular tongs 1-16. 


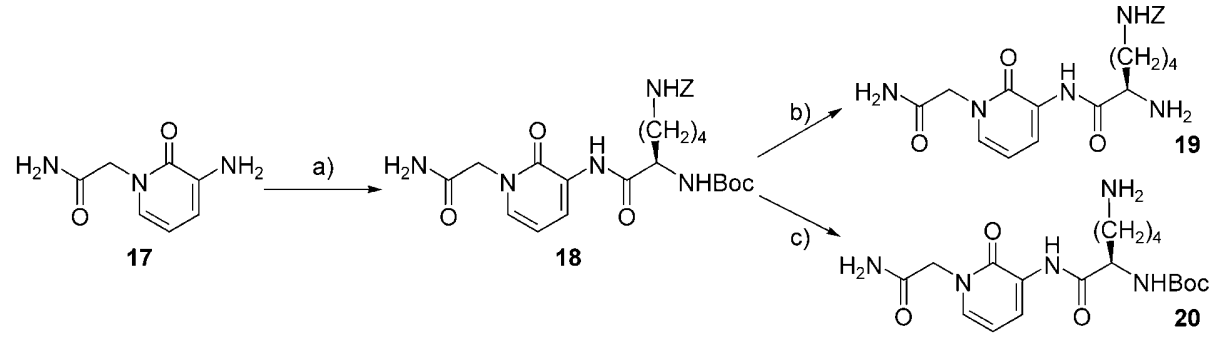

Scheme 1. Synthesis of peptidomimetic-containing strands 19 and 20. a) N-Boc-N-Z-K-OH, HBTU, HOBt, DIPEA, DMF, $48 \mathrm{~h}$, room temperature; b) TFA, $\mathrm{CH}_{2} \mathrm{Cl}_{2}, 2 \mathrm{~h}$, room temperature; c) $\mathrm{H}_{2}, 10 \% \mathrm{Pd} / \mathrm{C}, \mathrm{MeOH}, 12 \mathrm{~h}$, room temperature.

Protected peptidomimetic strand $\mathbf{2 2}$ was obtained from $\mathrm{N}$ (3-hydrazinocarbonyl-4-methoxyphenyl)acetamide $(21){ }^{[20]}$ following the same procedure as described for 18 (Scheme 2). Compound $\mathbf{2 2}$ was deprotected by acidic hydrolysis to give $\mathbf{2 3}$, or by hydrogenolysis to give $\mathbf{2 4}$, both in good yields (Scheme 2).
(Scheme 4). Cleavage of the $N$ Boc group of compounds 13 and $\mathbf{1 5}$ gave molecular tongs 14 and 16 , respectively, while hydrogenolysis of 9 and 11 afforded molecular tongs 10 and $\mathbf{1 2}$, respectively (Scheme 4).

\section{Biology}

The inhibitory activities of compounds 1-16 were assayed against recombinant wild-type PR (WT-PR) at pH 4.7 and $30{ }^{\circ} \mathrm{C}$ using a fluorimetric assay. ${ }^{[18-20]}$ The most efficient inhibitors were also assayed against the multidrug-resistant mutated protease ANAM-11. Zhang-Poorman kinetic analyses were used to characterize the mechanism of inhibition as dimerization alone (parallel lines), competitive inhibition (altered slopes and unaltered $y$-axis intercepts), and mixed inhibition (altered slopes and y-axis intercepts). The results are summarized in Table 1. Parallel lines were obtained for compounds 1-6, 8, and 15, demonstrating that they act purely as dimerization inhibitors (Figure 3 ). Compounds 7, 9, 11, and 13 exhibited mixed inhibition. Weak inhibitory effects were observed with compounds 10,12,14, and $16(28-30 \%$ at $14 \mu \mathrm{M}$ for 12 , or $28 \mu \mathrm{M}$ for 10,14 , and 16$)$.

Scheme 2. Synthesis of peptidomimetic-containing strands 23 and 24: a) N-Boc-N-Z-K-OH, HBTU, HOBt, DIPEA, DMF, $24 \mathrm{~h}$, room temperature; b) TFA, $\mathrm{CH}_{2} \mathrm{Cl}_{2}, 1 \mathrm{~h}$, room temperature; c) $\mathrm{H}_{2}, 10 \% \mathrm{Pd} / \mathrm{C}, \mathrm{MeOH}, 12 \mathrm{~h}$, room temperature.

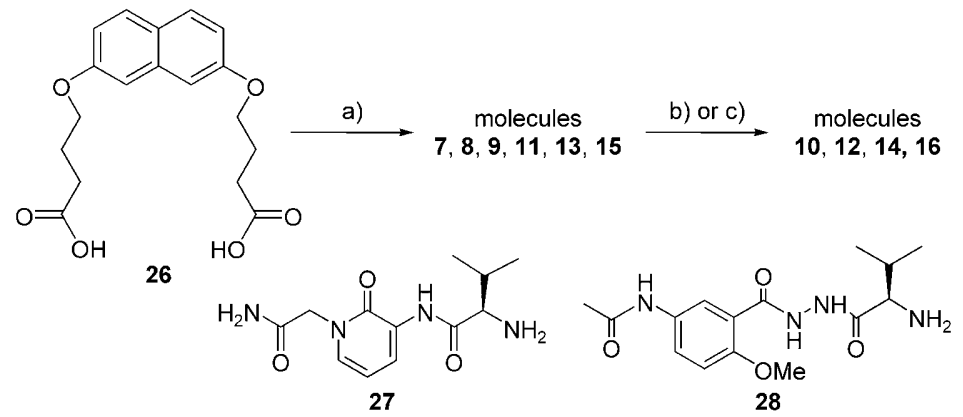

Scheme 4. Synthesis of molecular tongs 7-16. a) HBTU, HOBt, DIPEA, 19, 20, 23, 24, 27, or $28, \mathrm{DMF}, 48 \mathrm{~h}$, room temperature; b) for 14 or $16: 13$ or $15, \mathrm{TFA}, \mathrm{CH}_{2} \mathrm{Cl}_{2}, 30$ min, room temperature; c) for 10 or $12: 9$ or $11, \mathrm{H}_{2}, 10 \% \mathrm{Pd} / \mathrm{C}, \mathrm{MeOH} / \mathrm{DMF}, 12 \mathrm{~h}$, room temperature.

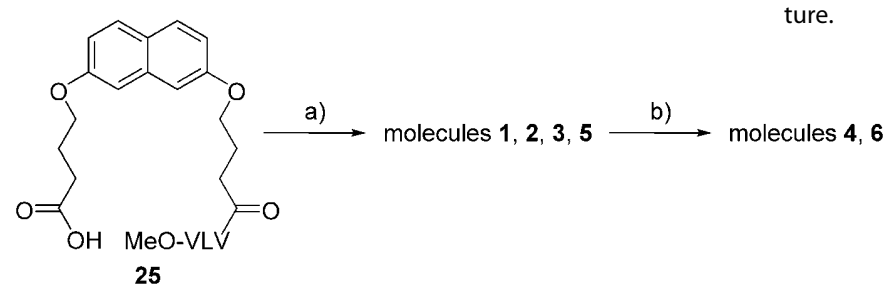

Scheme 3. Synthesis of molecular tongs 1-6. a) HBTU, HOBt, DIPEA, 19, 20, 23, or $\mathbf{2 4}, \mathrm{DMF}, 48 \mathrm{~h}$, room temperature; b) for 4 or $\mathbf{6 : 3}$ or $5, \mathrm{TFA}, \mathrm{CH}_{2} \mathrm{Cl}_{2}$, $30 \mathrm{~min}$, room temperature.
Based on the observed results, it was noted that replacement of the valine residue by an $\mathrm{N}$-protected lysine (asymmetrical molecular tongs 1 and 2) dramatically increases the inhibitory potency ( $40 \mathrm{~nm}$ for 1 relative to $400 \mathrm{~nm}$ for the valine analogue, ${ }^{[20]}$ and $60 \mathrm{~nm}$ for 2 relative to $200 \mathrm{~nm}$ for the valine analogue $\left.{ }^{[20]}\right)$. The symmetric molecular tongs 7,9 , and $\mathbf{1 1}$ are fairly efficient inhibitors that act through a mixed inhibition mechanism. For example, 11 exhibits both a dimerization inhibition 


\begin{tabular}{|c|c|c|c|}
\hline Compd & WT PR or ANAM-11 & $K_{\mathrm{ic}}[\mu \mathrm{M}]^{[\mathrm{a}]}$ & $K_{\mathrm{id}}[\mu \mathrm{M}]^{[\mathrm{bb}]}$ \\
\hline \multirow[t]{2}{*}{1} & WT & & 0.04 \\
\hline & ANAM-11 & & 0.25 \\
\hline \multirow[t]{2}{*}{2} & WT & & 0.06 \\
\hline & ANAM-11 & & 0.1 \\
\hline 3 & WT & & 1.2 \\
\hline 4 & WT & & 4.5 \\
\hline \multirow[t]{2}{*}{5} & WT & & 0.4 \\
\hline & ANAM-11 & & 1.36 \\
\hline 6 & WT & & 3 \\
\hline 7 & WT & 0.550 & 0.053 \\
\hline 8 & WT & & 0.220 \\
\hline 9 & WT & 0.2 & 0.12 \\
\hline 10 & WT & & $-^{[c]}$ \\
\hline 11 & WT & 0.15 & 0.053 \\
\hline 12 & WT & & $-^{[c]}$ \\
\hline 13 & WT & 31 & 17 \\
\hline 14 & WT & & $-^{[c]}$ \\
\hline \multirow[t]{2}{*}{15} & WT & & 0.28 \\
\hline & ANAM-11 & & 2 \\
\hline 16 & WT & & $-^{[c]}$ \\
\hline
\end{tabular}

[a] Competitive active site inhibition; [b] dimerization inhibition; [c] percent inhibition at $28 \mu \mathrm{M}: 28$ (10), 30 (14 and 16); at $14 \mu \mathrm{M}$ : 30 (12). Standard errors of initial rates are $<5 \%$.

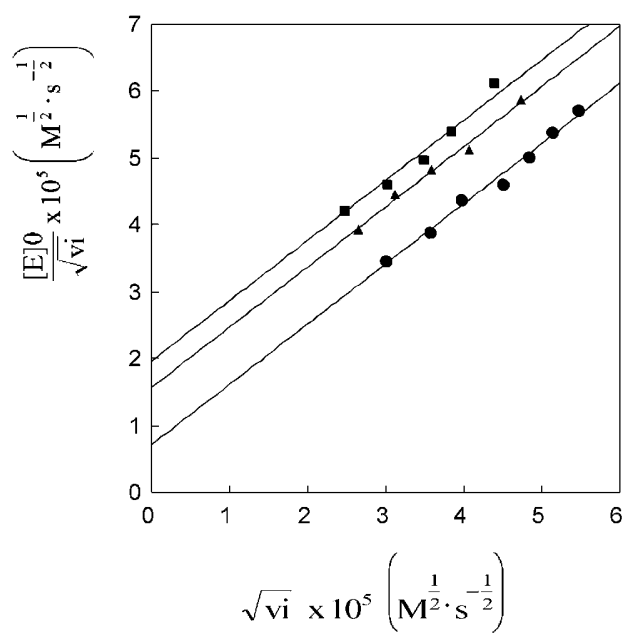

Figure 3. Plots of $[E]_{0} / \sqrt{ } v_{\mathrm{i}} v \mathrm{~V} \sqrt{ } v_{\mathrm{i}}$ for the hydrolysis of the fluorogenic substrate DABCYL- $\gamma$-abu-Ser-GIn-Asn-Tyr-Pro-lle-Val-Gln-EDANS by wild-type HIV-1 PR at pH 4.7 and $30^{\circ} \mathrm{C}$ in the absence ( $\bullet$ ) and presence of compound 15 at $9.25 \mu \mathrm{M}(\mathbf{-})$ and $7.08 \mu \mathrm{M}(\mathbf{\Delta})$.

component $\left(K_{\mathrm{id}}=53 \mathrm{~nm}\right)$ and a slight competitive inhibition component $\left(K_{\mathrm{ic}}=150 \mathrm{~nm}\right)$. Increasing both the length and flexibility of the nonpeptidic strand in asymmetrical molecular tong 3 has a detrimental effect on the inhibitory potency ( 3 vs. 1). In contrast, a simultaneous increase in both length and flexibility of the nonpeptidic strand in symmetrical molecular tong 15 has a favorable effect on inhibitory potency and results in purely dimerization inhibition activity (15 vs. 11) although the peptidic character of molecular tong $\mathbf{1 5}$ was completely suppressed. The hydrazide motif favors both dimerization inhibi- tion and inhibitory efficacy (15 vs 13). Deprotection of the amino moiety of the strands noticeably decreased the inhibitory potency ( 10 vs. 9,12 vs. 11,14 vs. 13 , and 16 vs. 15), confirming a favorable influence of the hydrophobic character of the tong arms on inhibitory activity. ${ }^{[18,19]}$ Remarkably, new pseudopeptidic molecular tongs 1, 2, 5, and 15 behaved as antidimers against the multimutated protease ANAM-11, which is analogous to a protease found in multi-drug resistant viruses. In contrast, the affinity of ritonavir for this mutated protease ${ }^{[21]}$ was about 78000-fold lower than for wild-type PR, demonstrating that the peptidomimetic molecular tongs 1, 2, 5, and 15 can still efficiently inhibit dimerization of mutated proteases. Compound 2 exhibits 20-fold higher in vitro activity toward ANAM-11 protease than does ritonavir. ${ }^{[21]}$ This confirms that inhibitors of PR dimerization are insensitive to protease mutations.

\section{Conclusions}

Molecular tongs, containing a naphthalene scaffold and peptidic side arms, were reported to be inhibitors of the PR interface. ${ }^{[18,19]}$ Replacement of two amino acids by peptidomimetics in one strand of these molecular tongs led to proteolysis-resistant inhibitors. ${ }^{[20]}$ We replaced the remaining valine residue by a more hydrophobic and flexible side chain of the Cbz-protected lysine residue to afford the most potent molecular tong inhibitor of PR dimerization $(\sim 40 \mathrm{~nm})$. In addition, replacement of two amino acids by peptidomimetics in both strands of these symmetrical molecular tongs predominantly resulted in a mixed mechanism of inhibition (competitive inhibition and antidimeric activity). The replacement of three amino acids by nonpeptidic fragments in each of two strands of the molecular tongs resulted in the first potent nonpeptidic protease inhibitor able to dissociate the mature protease dimer. In this way, we demonstrated that total suppression of peptidic character within this class of molecules does not alter their mechanism of action.

Four dimerization inhibitors were also evaluated against the ANAM-11 protease, which contains 11 mutations, and were found to be equally active toward both ANAM-11 and wildtype PR. We corroborated previous findings that peptidomimetic molecular tongs are candidates for successfully overcoming the resistance presently encountered with classical protease inhibitors. Deprotection of amino groups in the strands noticeably decreased the inhibitory potency of these molecules. Further studies of PR dimerization inhibitors containing peptidomimetics will focus on the synthesis of molecular tongs with greater hydrophilic character.

\section{Experimental Section}

\section{Synthesis}

Common solvents were purchased from commercial sources. $\mathrm{N}, \mathrm{N}$ Dimethylformamide (DMF) was distilled over $\mathrm{CaSO}_{4}$, tetrahydrofuran (THF) was distilled over sodium/benzophenone, and $\mathrm{CH}_{3} \mathrm{CN}$ was distilled over $\mathrm{CaCl}_{2}$. TLC was performed on silica gel $60 \mathrm{~F}_{250}$ $(0.26 \mathrm{~mm}$ thickness) plates. The plates were visualized with UV 
light $(\lambda 254 \mathrm{~nm})$, or with a $3.5 \%$ solution of phosphomolybdic acid or ninhydrin in EtOH. Liquid chromatography was performed on Merck 60 silica gel (230-400 mesh). Protected amino acids O-benzotriazol-1-yl- $N, N, N^{\prime}, N^{\prime}$-tetramethyluronium hexafluorophosphate (HBTU) and 1-hydroxybenzotriazol (HOBT) were purchased from commercial sources. H-Val-Leu-Val-OMe, ${ }^{[19]}$ 4-[7-(3-carboxypropoxy)naphtalen-2-yloxy]butyryl-Val-Leu-Val-OMe $\quad$ (25), ${ }^{[19]} \quad$ 2-(3-amino-2oxo-2H-pyridin-1-yl)acetamide $\quad(17){ }^{[20]} \quad \mathrm{N}$-(3-hydrazinocarbonyl-4methoxyphenyl)acetamide (21), ${ }^{[20]} 4-\{[7-(3-$ carboxypropoxy)-2-naphtyl]oxy\}butanoic acid (26), ${ }^{[18]}$ 2-amino- $N$-(1-carbamoylmethyl-2-oxo1,2-dihydropyridin-3-yl)-3-methylbutyramide (27) ${ }^{[20]}$ and $N$-\{3-[N'-(2amino-3-methylbutyryl)hydrazinocarbonyl]-4-methoxyphenyl\}acetamide $(\mathbf{2 8})^{[20]}$ were prepared according to published methods. Melting points were determined on a Kofler melting point apparatus. NMR spectra were performed on a Ultrafield AVANCE $300\left({ }^{1} \mathrm{H}\right.$, $\left.300 \mathrm{MHz} ;{ }^{13} \mathrm{C}, 75 \mathrm{MHz}\right)$ or a Bruker AVANCE $400\left({ }^{1} \mathrm{H}, 400 \mathrm{MHz} ;{ }^{13} \mathrm{C}\right.$, $100 \mathrm{MHz}$ ). Unless otherwise stated, $\mathrm{CDCl}_{3}$ was used as solvent. Chemical shifts $(\delta)$ are in ppm, using the following abbreviations: singlet $(\mathrm{s})$, doublet $(\mathrm{d})$, doublet doublet $(\mathrm{dd})$, triplet $(\mathrm{t})$, quintuplet (quint), multiplet (m), broad multiplet (bm), and broad singlet (bs). Mass spectra were obtained using a Bruker Esquire electrospray ionization apparatus at the SAMM (Faculty of Pharmacy at Châtenay-Malabry, France). Elemental analyses $(C, H, N)$ were performed on a PerkinElmer CHN Analyser 2400 at the Microanalyses Service of the Faculty of Pharmacy at Châtenay-Malabry (France). Elemental analysis data are included in the Supporting Information.

[5-Benzyloxycarbonylamino-5-(1-carbamoylmethyl-2-oxo-1,2-dihydropyridin-3-ylcarbamoyl)pentyl]carbamic acid tert-butyl ester (18). Compound $17(436 \mathrm{mg}, 2.61 \mathrm{mmol})$ and $N_{\alpha}$-Boc- $N_{\varepsilon}-Z$-Lys $(1.09 \mathrm{~g}, 2.87 \mathrm{mmol})$ were dissolved in DMF $(20 \mathrm{~mL})$. DIPEA $(1.1 \mathrm{~mL}$, $6.53 \mathrm{mmol})$, HBTU $(1.19 \mathrm{~g}, 3.13 \mathrm{mmol})$, and HOBt $(388 \mathrm{mg}$, $2.87 \mathrm{mmol}$ ) were successively added to the reaction mixture. The reaction mixture was stirred under argon at room temperature for $48 \mathrm{~h}$. DMF was evaporated under reduced pressure and the residue was dissolved in EtOAc $(200 \mathrm{~mL})$. The organic phase was washed with $10 \%$ aqueous citric acid $(50 \mathrm{~mL}), \mathrm{H}_{2} \mathrm{O}(100 \mathrm{~mL}), 10 \%$ aqueous $\mathrm{K}_{2} \mathrm{CO}_{3}(50 \mathrm{~mL})$, and brine $(25 \mathrm{~mL})$. The organic phase was dried over $\mathrm{Na}_{2} \mathrm{SO}_{4}$, filtered and concentrated under reduced pressure. The resulting crude product was purified by flash chromatography on silica gel and eluted with EtOAc/MeOH (95:5) to give 18 as a

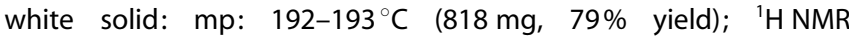
([D $\left.\left.\mathrm{D}_{6}\right] \mathrm{DMSO}\right): \delta=9.1(\mathrm{~s}, 1 \mathrm{H}), 8.2(\mathrm{dd}, J=1.3,7.3 \mathrm{~Hz}, 1 \mathrm{H}), 7.6(\mathrm{~s}, 1 \mathrm{H})$, 7.6-7.3 (m, 6H,), $7.2(\mathrm{~s}, 1 \mathrm{H}), 6.2(\mathrm{t}, J=7.3 \mathrm{~Hz}, 1 \mathrm{H}), 5.0(\mathrm{~s}, 2 \mathrm{H}), 4.6(\mathrm{~s}$, $2 \mathrm{H}), 4.0(\mathrm{~m}, 1 \mathrm{H}), 3.0(\mathrm{q}, J=6 \mathrm{~Hz}, 2 \mathrm{H}), 1.7(\mathrm{~m}, 1 \mathrm{H}), 1.6(\mathrm{~m}, 1 \mathrm{H}), 1.4-$ $1.3(\mathrm{~m}, 13 \mathrm{H}) ;{ }^{13} \mathrm{C}$ NMR ([D $]$ DMSO): $\delta=171.8,168.3,156.7,156.1$, $155.7,137.3,133.0,128.3,128.0,127.7,122.0,104.7,78.5,65.1,55.4$, 51.2, 40.1, 30.8, 29.0, 28.1, 22.9; ESI ${ }^{+} \mathrm{MS} \mathrm{m} / \mathrm{z}: 553[\mathrm{M}+\mathrm{Na}]^{+}, 568$ $[M+39]^{+}$; Anal. $(\mathrm{C}, \mathrm{H}, \mathrm{N}): \mathrm{C}_{26} \mathrm{H}_{35} \mathrm{~N}_{5} \mathrm{O}_{7}$.

[5-Amino-5-(1-carbamoylmethyl-2-oxo-1,2-dihydropyridin-3-ylcarbamoyl)pentyl]carbamic acid benzyl ester in trifluoroacetic acid (19). Trifluoroacetic acid (TFA; $5 \mathrm{~mL}$ ) was added to a solution of 18 (300 mg, $0.57 \mathrm{mmol})$ in dry $\mathrm{CH}_{2} \mathrm{Cl}_{2}(5 \mathrm{~mL})$. The mixture was stirred at room temperature for $1.5 \mathrm{~h}$, then the solvent was evaporated under reduced pressure. The crude product was crystallized from $\mathrm{MeOH} / \mathrm{Et}_{2} \mathrm{O}$ to yield 19 as a beige solid: $\mathrm{mp}$ : $124-126^{\circ} \mathrm{C}$ (248 mg, 100\% yield); ${ }^{1} \mathrm{H}$ NMR ([D $\left.\left.\mathrm{D}_{6}\right] \mathrm{DMSO}\right): \delta=10.0(\mathrm{~s}, 1 \mathrm{H}), 8.3-8.2$ $(\mathrm{m}, 4 \mathrm{H}), 7.6(\mathrm{~s}, 1 \mathrm{H}), 7.4-7.2(\mathrm{~m}, 7 \mathrm{H}), 6.3(\mathrm{t}, J=7.2 \mathrm{~Hz}, 1 \mathrm{H}), 5.0(\mathrm{~s}$, $2 \mathrm{H}), 4.6(\mathrm{~s}, 2 \mathrm{H}), 4.26(\mathrm{~m}, 1 \mathrm{H}), 2.98(\mathrm{~m}, 2 \mathrm{H}), 1.74(\mathrm{~m}, 2 \mathrm{H})$ 1.4-1.3 (m, $4 \mathrm{H}) ;{ }^{13} \mathrm{C}$ NMR $\left(\mathrm{CD}_{3} \mathrm{OD}\right) \delta=171.4,169.2,159.15,156.5,135.1,129.5$, 129.3, 129.0, 128.8, 127.3, 107.3, 67.4, 55.0, 52.9, 41.2, 32.4, 30.5, 23.0; $\mathrm{ESI}^{+} \mathrm{MS} \mathrm{m} / \mathrm{z}: 430[\mathrm{M+H}]^{+}$; HRMS calcd for $\mathrm{C}_{21} \mathrm{H}_{27} \mathrm{~N}_{5} \mathrm{O}_{5}+\mathrm{H}$ : 430.2090, found: 430.2112 .
[5-Amino-5-(1-carbamoylmethyl-2-oxo-1,2-dihydropyridin-3-ylcarbamoyl)pentyl]carbamic acid tert-butyl ester (20). Pd/C $10 \%$ (53 mg, 20\% mass) was added to a solution of 18 (264 mg, $0.5 \mathrm{mmol})$ in $\mathrm{MeOH}(25 \mathrm{~mL})$. The reaction flask was purged three times with hydrogen, and stirring was maintained under hydrogen atmosphere at room temperature for $12 \mathrm{~h}$. After filtration through Celite, the cake was washed with $\mathrm{MeOH}(200 \mathrm{~mL})$, and the filtrate was concentrated to afford a solid that was triturated with cyclohexane and petroleum ether to yield 20 as a white solid: $\mathrm{mp}: 146-$ $148^{\circ} \mathrm{C}(190 \mathrm{mg}, 96 \%$ yield $) ;{ }^{1} \mathrm{H}$ NMR: $\delta=9.2(\mathrm{~s}, 1 \mathrm{H}), 8.4(\mathrm{~d}, J=$ $7.3 \mathrm{~Hz}, 1 \mathrm{H}), 7.6(\mathrm{~s}, 1 \mathrm{H}), 7.1(\mathrm{~d}, J=7.3 \mathrm{~Hz}, 1 \mathrm{H}), 6.9(\mathrm{~s}, 1 \mathrm{H}), 5.9$ (bs, $1 \mathrm{H}), 5.4(\mathrm{bs}, 1 \mathrm{H}), 6.2(\mathrm{t}, J=7.3 \mathrm{~Hz}, 1 \mathrm{H}), 4.6(\mathrm{~s}, 2 \mathrm{H}), 4.4(\mathrm{~m}, 1 \mathrm{H}), 2.7$ $(\mathrm{m}, 2 \mathrm{H}), 1.9(\mathrm{~m}, 1 \mathrm{H}), 1.7(\mathrm{~m}, 1 \mathrm{H}), 1.4-1.2(\mathrm{~m}, 13 \mathrm{H}) ;{ }^{13} \mathrm{C}$ NMR: $\delta=$ $171.7,168.9,156.6,156.2,131.3,127.8,123.2,107.3,78.4,54.0,53.3$, 41.4, 32.4, 30.0, 28.4, 22.8; ESI ${ }^{+}$MS m/z: $418[\mathrm{M}+\mathrm{Na}]^{+}$; HRMS calcd for $\mathrm{C}_{18} \mathrm{H}_{29} \mathrm{~N}_{5} \mathrm{O}_{5}+\mathrm{Na}: 418.2066$, found: 418.2078 .

\{6-[N'-(5-Acetylamino-2-methoxybenzoyl)hydrazino]-5-benzyloxycarbonylamino-6-oxohexyl\}carbamic acid tert-butyl ester (22). Compound 22 was synthesized from 21, following the same procedure described for $\mathbf{1 8}$, and was obtained as a white solid in $73 \%$ yield: $\mathrm{mp}:{ }^{136}-138^{\circ} \mathrm{C}$; ${ }^{1} \mathrm{H}$ NMR: $\delta=11.9(\mathrm{~s}, 1 \mathrm{H}), 11.3(\mathrm{~d}, J=$ $7.0 \mathrm{~Hz}, 1 \mathrm{H}), 8.8(\mathrm{~s}, 1 \mathrm{H}), 8.4(\mathrm{~d}, J=9.0 \mathrm{~Hz}, 1 \mathrm{H}), 7.9(\mathrm{~s}, 1 \mathrm{H}), 7.3(\mathrm{~m}$, $5 \mathrm{H}), 7.0(\mathrm{~d}, J=9.0 \mathrm{~Hz}, 1 \mathrm{H}), 5.6(\mathrm{~d}, J=7.5 \mathrm{~Hz}, 1 \mathrm{H}), 5.0(\mathrm{~s}, 2 \mathrm{H}), 4.9(\mathrm{~m}$, $1 \mathrm{H}), 4.7(\mathrm{~s}, 1 \mathrm{H}), 4.0(\mathrm{~s}, 3 \mathrm{H}), 3.0(\mathrm{~m}, 2 \mathrm{H}), 2.2(\mathrm{~s}, 3 \mathrm{H}), 1.8(\mathrm{~m}, 1 \mathrm{H}), 1.7$ $(\mathrm{m}, 1 \mathrm{H}), 1.4(\mathrm{~s}, 9 \mathrm{H}), 1.4-1.3(\mathrm{~m}, 4 \mathrm{H}) ;{ }^{13} \mathrm{C}$ NMR: $\delta=169.0,166.3$, 158.6, 156.3, 155.6, 153.6, 136.6, 133.1, 128.4, 128.0, 126.1, 122.5, $117.8,112.4,79.8,66.5,56.5,52.0,40.7,34.0,29.5,28.6,24.6,21.9$; $\mathrm{ESI}^{+} \mathrm{MS} \mathrm{m} / \mathrm{z}: 608[\mathrm{M+Na}]^{+}$; Anal. $(\mathrm{C}, \mathrm{H}, \mathrm{N}): \mathrm{C}_{29} \mathrm{H}_{39} \mathrm{~N}_{5} \mathrm{O}_{8}$.

\{6-[N'-(5-Acetylamino-2-methoxybenzoyl)hydrazino]-5-amino-6oxohexyl\}carbamic acid benzyl ester (23). TFA (4 mL) was added to a solution of $22(350 \mathrm{mg}, 0.60 \mathrm{mmol})$ in dry $\mathrm{CH}_{2} \mathrm{Cl}_{2}(4 \mathrm{~mL})$. The mixture stirred at room temperature for $1 \mathrm{~h}$, then the solvent was evaporated under reduced pressure. The crude product was crystallized from $\mathrm{MeOH} / \mathrm{Et}_{2} \mathrm{O}$ to yield compound 23 as a light pink solid: $\mathrm{mp}: 140-142^{\circ} \mathrm{C}\left(315 \mathrm{mg}, 88 \%\right.$ yield); ${ }^{1} \mathrm{H}$ NMR ([D $]$ DMSO): $\delta=10.9(\mathrm{~s}, 1 \mathrm{H}), 10.2(\mathrm{~s}, 1 \mathrm{H}), 10.0(\mathrm{~s}, 1 \mathrm{H}), 8.2(\mathrm{bs}, 2 \mathrm{H}), 8.0(\mathrm{~s}, 1 \mathrm{H})$, $7.7(\mathrm{~d}, J=9.0 \mathrm{~Hz}, 1 \mathrm{H}), 7.4-7.3(\mathrm{~m}, 6 \mathrm{H}), 7.1(\mathrm{~d}, J=9.0 \mathrm{~Hz}, 1 \mathrm{H}), 5.0(\mathrm{~s}$, $2 \mathrm{H}), 3.8\left(\mathrm{~m}, 4 \mathrm{H}, \mathrm{H}_{11}\right), 3.0(\mathrm{~m}, 2 \mathrm{H}), 2.0(\mathrm{~s}, 3 \mathrm{H}), 1.8(\mathrm{~m}, 2 \mathrm{H}), 1.5-1.4$ $(\mathrm{m}, 4 \mathrm{H}) ;{ }^{13} \mathrm{C}$ NMR $\left(\left[\mathrm{D}_{4}\right] \mathrm{MeOH}\right): \delta=171.6,169.1,158.6,166.4,159.1$, $155.7,133.5,129.5,129.0,128.7,127.0,124.4,120.9,113.5,67.4$, 56.9, 53.4, 41.2, 32.2, 30.5, 23.6, 22.9; $\mathrm{APCl}^{+} \mathrm{MS} \mathrm{m} / \mathrm{z}: 487[\mathrm{M+H}]^{+}$; Anal. $(\mathrm{C}, \mathrm{H}, \mathrm{N}): \mathrm{C}_{26} \mathrm{H}_{32} \mathrm{~N}_{5} \mathrm{O}_{6} \cdot \mathrm{CF}_{3} \mathrm{COOH} \cdot 1.5 \mathrm{H}_{2} \mathrm{O}$.

\{6-[ $N^{\prime}$-(5-Acetylamino-2-methoxybenzoyl)hydrazino]5-amino-6oxohexyl\}carbamic acid tert-butyl ester (24). Pd/C $10 \%(23 \mathrm{mg}$, $20 \%$ mass) was added to a solution of $22(150 \mathrm{mg}, 0.26 \mathrm{mmol})$ in $\mathrm{MeOH}(15 \mathrm{~mL})$. The reaction flask was purged three times with hydrogen, and stirring was maintained under hydrogen atmosphere at room temperature for $12 \mathrm{~h}$. The mixture was filtered through celite, and the cake was washed with $\mathrm{MeOH}(200 \mathrm{~mL})$. The filtrate was evaporated to dryness to yield crude product 24 as a colorless oil $\left(111 \mathrm{mg}, 96 \%\right.$ yield): ${ }^{1} \mathrm{H}$ NMR: $\delta=9.3(\mathrm{~s}, 1 \mathrm{H}), 8.9(\mathrm{~s}, 1 \mathrm{H}), 8.3(\mathrm{~s}$, $2 \mathrm{H}), 7.9(\mathrm{~m}, 1 \mathrm{H}), 6.9(\mathrm{~m}, 1 \mathrm{H}), 5.6(\mathrm{~m}, 1 \mathrm{H}), 4.6(\mathrm{~m}, 2 \mathrm{H}), 4.1-3.9(\mathrm{~m}$, $4 \mathrm{H}), 2.3-2.2(\mathrm{~m}, 5 \mathrm{H}), 1.8(\mathrm{bm}, 2 \mathrm{H}), 1.5-1.3(\mathrm{~m}, 13 \mathrm{H}) ;{ }^{13} \mathrm{C}$ NMR: $\delta=$ $169.2,169.1,158.5,155.6,153.7,132.9,125.9,122.7,118.2,112.0$, 79.9, 56.47, 52.3, 52.2, 50.2, 27.0, 28.4, 24.4, 22.2; ESI ${ }^{+} \mathrm{MS} \mathrm{m} / \mathrm{z}: 452$ $[\mathrm{M}+\mathrm{H}]^{+}$; HRMS calcd for $\mathrm{C}_{21} \mathrm{H}_{33} \mathrm{~N}_{5} \mathrm{O}_{6}+\mathrm{H}: 452.2509$, found: 452.2526 .

Synthesis of molecular tong 1. Compounds 25 (200 mg, $0.3 \mathrm{mmol})$ and $19(195 \mathrm{mg}, 0.36 \mathrm{mmol})$ were dissolved in DMF $(10 \mathrm{~mL})$. DIPEA $(0.37 \mathrm{~mL}, 2.1 \mathrm{mmol})$, HBTU $(284 \mathrm{mg}, 0.75 \mathrm{mmol})$, and HOBt ( $89 \mathrm{mg}, 0.66 \mathrm{mmol}$ ) were successively added to the reaction mixture, which stirred under argon at room temperature for 
$48 \mathrm{~h}$. DMF was evaporated under reduced pressure, and the residue was washed successively with EtOAc, $\mathrm{CH}_{2} \mathrm{Cl}_{2}, \mathrm{MeOH}, \mathrm{H}_{2} \mathrm{O}$ $(100 \mathrm{~mL}), 10 \%$ aqueous citric acid $(50 \mathrm{~mL}), 10 \%$ aqueous $\mathrm{K}_{2} \mathrm{CO}_{3}$ $(50 \mathrm{~mL})$, and $\mathrm{H}_{2} \mathrm{O}$ again $(50 \mathrm{~mL})$. Product 1 was obtained as a white solid: $\mathrm{mp}: 233-235^{\circ} \mathrm{C}(277 \mathrm{mg}, 86 \%) ;{ }^{1} \mathrm{H}$ NMR ([D 6 DMSO): $\delta=9.2$ (s, $1 \mathrm{H}), 8.3(\mathrm{~d}, J=7.3 \mathrm{~Hz}, 1 \mathrm{H}), 8.2(\mathrm{~d}, J=7.2 \mathrm{~Hz}, 1 \mathrm{H}), 8.0-7.9(\mathrm{~m}$, $3 \mathrm{H}), 7.7(\mathrm{~d}, J=8.9 \mathrm{~Hz}, 2 \mathrm{H}), 7.6(\mathrm{~s}, 1 \mathrm{H}), 7.3-7.1(\mathrm{~m}, 6 \mathrm{H}), 7.2(\mathrm{~s}, 2 \mathrm{H})$, $7.1(\mathrm{~s}, 2 \mathrm{H}), 7.0(\mathrm{~d}, J=8.9 \mathrm{~Hz}, 2 \mathrm{H}), 6.2(\mathrm{t}, J=7.2 \mathrm{~Hz}, 1 \mathrm{H}), 5.0(\mathrm{~s}, 2 \mathrm{H})$ $4.5(\mathrm{~s}, 2 \mathrm{H}), 4.4-4.3(\mathrm{~m}, 2 \mathrm{H}), 4.1(\mathrm{q}, J=7.2 \mathrm{~Hz}, 2 \mathrm{H}), 4.0(\mathrm{q}, J=6.5 \mathrm{~Hz}$, $4 \mathrm{H}), 3.6(\mathrm{~s}, 3 \mathrm{H}), 2.9(\mathrm{q}, J=6.1 \mathrm{~Hz}, 2 \mathrm{H}), 2.4-2.3(\mathrm{~m}, 4 \mathrm{H}), 2.2-2.0(\mathrm{~m}$, $6 \mathrm{H}), 1.8-1.7(\mathrm{~m}, 1 \mathrm{H}), 1.6-1.5(\mathrm{~m}, 2 \mathrm{H}), 1.5-1.3(\mathrm{~m}, 4 \mathrm{H}), 1.3(\mathrm{~m}, 2 \mathrm{H})$, $0.9-0.8(\mathrm{~m}, 18 \mathrm{H}) ;{ }^{13} \mathrm{C}$ NMR ([D $]$ DMSO): $\delta=172.3,172.2,171.7$, $171.3,170.9,168.3,157.0,156.7,156.1,137.3,135.7,133.1,128.9$, $128.3,128.1,127.7,123.7,122.3,115.9,106.1,104.7,66.9,65.1,57.8$, $57.3,53.7,51.6,51.3,50.8,40.7,40.1,31.6,30.7,30.3,29.9,29.1$, 25.0, 24.9, 24.1, 22.9, 22.8, 21.6, 19.2, 18.8, 18.2, 18.1; $\mathrm{ESI}^{+} \mathrm{MS} \mathrm{m} / \mathrm{z}$ : $1092[\mathrm{M}+\mathrm{Na}]^{+}, 1108[M+\mathrm{K}]^{+}$; HRMS (calcd for $\mathrm{C}_{56} \mathrm{H}_{76} \mathrm{~N}_{8} \mathrm{O}_{13}+\mathrm{Na}$ : 1092.2376, found: 1091.5454; Anal. (C, H, N): $\mathrm{C}_{56} \mathrm{H}_{76} \mathrm{~N}_{8} \mathrm{O}_{13}$.

Synthesis of molecular tong 2 . Tong 2 was synthesized from 23, following the same procedure as described for 1 from 25, and was obtained as a white solid in $63 \%$ yield: $\mathrm{mp}$ : $199-201{ }^{\circ} \mathrm{C} ;{ }^{1} \mathrm{H}$ NMR ([D $\left.\left.\mathrm{D}_{6}\right] \mathrm{DMSO}\right): \delta=10.3$ (bs, $\left.1 \mathrm{H}\right), 10.1(\mathrm{bs}, 1 \mathrm{H}), 9.9(\mathrm{~s}, 1 \mathrm{H}), 8.0(\mathrm{~d}, J=$ $7.1 \mathrm{~Hz}, 1 \mathrm{H}), 8.0-7.9(\mathrm{~m}, 4 \mathrm{H}), 7.76(\mathrm{dd}, J=2.4,9.0 \mathrm{~Hz}, 1 \mathrm{H}), 7.7(\mathrm{~d}, J=$ $8.8 \mathrm{~Hz}, 2 \mathrm{H}), 7.4-7.3(\mathrm{~m}, 5 \mathrm{H}), 7.2(\mathrm{t}, J=5.4 \mathrm{~Hz}, 1 \mathrm{H}), 7.2(\mathrm{~m}, 2 \mathrm{H}), 7.1$ $(\mathrm{d}, J=9.0 \mathrm{~Hz}, 1 \mathrm{H}), 7.0(\mathrm{~m}, 2 \mathrm{H}), 5.0(\mathrm{~s}, 2 \mathrm{H}), 4.4(\mathrm{q}, J=7.8 \mathrm{~Hz}, 2 \mathrm{H})$, $4.16(\mathrm{q}, J=7.8 \mathrm{~Hz}, 2 \mathrm{H}), 4.04(\mathrm{~m}, 4 \mathrm{H}), 3.84(\mathrm{~s}, 3 \mathrm{H}), 3.60(\mathrm{~s}, 3 \mathrm{H}), 3.0$ (q, $J=6.2 \mathrm{~Hz}, 2 \mathrm{H}), 2.3(\mathrm{t}, J=7.0 \mathrm{~Hz}, 4 \mathrm{H}), 2.0-1.9(\mathrm{~m}, 9 \mathrm{H}), 1.7(\mathrm{~m}$, $1 \mathrm{H}), 1.6(\mathrm{~m}, 2 \mathrm{H}), 1.5-1.4(\mathrm{~m}, 4 \mathrm{H}), 1.3(\mathrm{~m}, 2 \mathrm{H}), 0.9-0.8(\mathrm{~m}, 18 \mathrm{H})$; ${ }^{13} \mathrm{C}$ NMR ([D $]$ DMSO): $\delta=172.2,171.7,171.5,170.9,168.0,163.3$, 157.0, 156.1, 152.6, 137.3, 135.8, 132.6, 129.0, 128.3, 127.7, 123.7, $123.2,121.3,121.2,115.9,112.4,106.1,67.0,66.9,65.1,57.8,57.3$ $56.1,51.6,51.1,50.8,40.7,40.1,32.1,31.6,30.2,29.9,29.2,25.0$, $24.9,24.1,23.8,22.9,22.6,21.6,19.2,18.8,18.2,18.1$; ESI ${ }^{+}$MS m/z: $1148[\mathrm{M}+\mathrm{Na}]^{+}, 1164[\mathrm{M}+\mathrm{K}]^{+}$; Anal. $(\mathrm{C}, \mathrm{H}, \mathrm{N}): \mathrm{C}_{59} \mathrm{H}_{80} \mathrm{~N}_{8} \mathrm{O}_{14}$

Synthesis of molecular tong $\mathbf{3}$. Tong $\mathbf{3}$ was synthesized from $\mathbf{2 0}$ following the same procedure as described for 1 from 25, and was obtained as a white solid in $57 \%$ yield: $\mathrm{mp}$ : $172-176^{\circ} \mathrm{C}$; ${ }^{1} \mathrm{H}$ NMR ([D $\left.\left.\mathrm{D}_{6}\right] \mathrm{DMSO}\right): \delta=9.2(\mathrm{~s}, 1 \mathrm{H}), 8.2(\mathrm{~d}, J=7.5,1 \mathrm{H}), 7.9(\mathrm{~m}, 3 \mathrm{H}), 7.8(\mathrm{~s}$, $1 \mathrm{H}), 7.7(\mathrm{~d}, J=8.8,2 \mathrm{H}), 7.6(\mathrm{~s}, 1 \mathrm{H}), 7.4(\mathrm{~d}, J=6.4,1 \mathrm{H}), 7.3(\mathrm{~d}, J=$ 7.0, 1H), $7.2(\mathrm{~m}, 3 \mathrm{H}), 7.0(\mathrm{~d}, J=9.0,2 \mathrm{H}), 6.2(\mathrm{t}, J=6.7,1 \mathrm{H}), 4.6(\mathrm{~s}$, $2 \mathrm{H}), 4.38(\mathrm{~m}, 1 \mathrm{H}), 4.2-4.1(\mathrm{~m}, 2 \mathrm{H}), 4.1-4.0(\mathrm{~m}, 5 \mathrm{H}), 3.6(\mathrm{~s}, 3 \mathrm{H}), 3.0$ $(\mathrm{m}, 2 \mathrm{H}), 2.4-2.2(\mathrm{~m}, 4 \mathrm{H}), 2.0-1.9(\mathrm{~m}, 6 \mathrm{H}), 1.7-1.6(\mathrm{~m}, 1 \mathrm{H}), 1.6-1.5$ $(\mathrm{m}, 2 \mathrm{H}), 1.4-1.2(\mathrm{~m}, 15 \mathrm{H}), 0.9-0.7(\mathrm{~m}, 18 \mathrm{H}) ;{ }^{13} \mathrm{C}$ NMR ([D $\left.\left.\mathrm{D}_{6}\right] \mathrm{DMSO}\right)$ : $\delta=172.6,172.3,172.1,171.9,171.4,168.7,157.5,157.2,136.2$, 133.4, 129.4, 128.5, 124.2, 122.5, 116.4, 106.7, 105.2, 79.0, 67.4, 58.3, $57.7,55.9,52.0,51.7,51.3,41.2,38.7,32.3,32.1,31.2,30.6,30.3$, 29.2, 28.6, 25.5, 25.4, 24.5, 23.5, 23.4, 22.1, 19.6, 19.3, 18.6; ESI ${ }^{-} \mathrm{MS}$ $\mathrm{m} / \mathrm{z} 1034[\mathrm{M}-\mathrm{H}]^{+}$; Anal. $\left(\mathrm{C}_{53} \mathrm{H}_{78} \mathrm{~N}_{8} \mathrm{O}_{13} \cdot 3 \mathrm{H}_{2} \mathrm{O}\right) \mathrm{C}, \mathrm{H}, \mathrm{N}$.

Synthesis of molecular tong 4. TFA ( $2 \mathrm{~mL}, 26 \mathrm{mmol}$ ) was added to a solution of $3(100 \mathrm{mg}, 0.1 \mathrm{mmol})$ in dry $\mathrm{CH}_{2} \mathrm{Cl}_{2}(2 \mathrm{~mL})$. The mixture was stirred for $30 \mathrm{~min}$, then the solvent was evaporated under reduced pressure. Azeotropic removal of excess TFA was carried out using toluene. The crude product was recrystallized from $\mathrm{MeOH} / \mathrm{Et}_{2} \mathrm{O}$ to give 4 as a white solid: $\mathrm{mp}$ : $180-182{ }^{\circ} \mathrm{C}(100 \mathrm{mg}$, $99 \%) ;{ }^{1} \mathrm{H}$ NMR ([D $]$ DMSO): $\delta=10.0(\mathrm{~s}, 1 \mathrm{H}), 8.2(\mathrm{~d}, J=7.2,1 \mathrm{H}), 8.0-$ $7.8(\mathrm{~m}, 5 \mathrm{H}), 7.7(\mathrm{~d}, J=8.9,2 \mathrm{H}), 7.6(\mathrm{~s}, 1 \mathrm{H}), 7.4(\mathrm{~d}, J=6.8,1 \mathrm{H}), 7.2$ $(\mathrm{m}, 3 \mathrm{H}), 7.0(\mathrm{~m}, 1 \mathrm{H}), 6.3(\mathrm{t}, J=7.1,1 \mathrm{H}), 4.6(\mathrm{~s}, 2 \mathrm{H}), 4.4(\mathrm{~m}, 1 \mathrm{H}), 4.2-$ $4.0(\mathrm{~m}, 7 \mathrm{H}), 3.6(\mathrm{~s}, 3 \mathrm{H}), 3.1(\mathrm{~s}, 2 \mathrm{H}), 2.4-2.2(\mathrm{~m}, 4 \mathrm{H}), 2.0-1.8(\mathrm{~m}, 6 \mathrm{H})$, $1.8-1.6(\mathrm{~m}, 3 \mathrm{H}), 1.6-1.4(\mathrm{~m}, 6 \mathrm{H}), 0.9-0.8(\mathrm{~m}, 18 \mathrm{H}) ;{ }^{13} \mathrm{C} N M R$ ([D $\left.\left.\mathrm{D}_{6}\right] \mathrm{DMSO}\right): \delta=172.6,172.2,172.1,171.8,171.4,168.7,157.5$, $157.2,136.2,134.5,129.4,128.2,124.2,122.5,116.3,106.7,104.9$, $67.4,58.4,57.7,53.4,52.0,51.7,51.4,41.2,38.6,32.3,32.1,31.9$,
30.6, 30.3, 29.2, 25.5, 25.4, 24.5, 23.4, 22.3, 22.1, 19.6, 19.3, 18.6; $\mathrm{ESI}^{+} \mathrm{MS} \mathrm{m} / \mathrm{z}$ : $936[\mathrm{M}+\mathrm{H}]^{+}$, $953[\mathrm{M}+\mathrm{Na}]^{+}$; Anal. $(\mathrm{C}, \mathrm{H}, \mathrm{N})$ : $\mathrm{C}_{48} \mathrm{H}_{70} \mathrm{~N}_{8} \mathrm{O}_{11} \cdot \mathrm{CF}_{3} \mathrm{COOH}$.

Synthesis of molecular tong $\mathbf{5}$. Tong $\mathbf{5}$ was synthesized from 24, following the same procedure as described for 1 from 25 , and was obtained as a white solid in $53 \%$ yield: $\mathrm{mp}: 150-156^{\circ} \mathrm{C}$; ${ }^{1} \mathrm{H}$ NMR: $\delta=11.0$ (bs, $1 \mathrm{H}), 9.2(\mathrm{bs}, 1 \mathrm{H}), 8.5(\mathrm{~d}, J=9 \mathrm{~Hz}, 1 \mathrm{H}), 8.4$ (bs, $1 \mathrm{H}), 8.0$ $(\mathrm{s}, 1 \mathrm{H}), 7.9$ (bs, $1 \mathrm{H},), 7.58(\mathrm{~d}, J=8.8 \mathrm{~Hz}, 2 \mathrm{H}), 7.2$ (bs, 1H), 7.2-6.9 $(\mathrm{m}, 5 \mathrm{H}), 6.1-5.8(\mathrm{~m}, 2 \mathrm{H}), 4.8(\mathrm{~m}, 1 \mathrm{H}), 4.7(\mathrm{~m}, 1 \mathrm{H}), 4.6(\mathrm{~m}, 1 \mathrm{H}), 4.5$ $(\mathrm{m}, 1 \mathrm{H}), 4.1(\mathrm{~m}, 4 \mathrm{H}), 4.0(\mathrm{~s}, 3 \mathrm{H}), 3.7(\mathrm{~s}, 3 \mathrm{H}), 3.1(\mathrm{~m}, 2 \mathrm{H}), 2.6(\mathrm{~m}$ $1 \mathrm{H}), 2.4(\mathrm{~m}, 4 \mathrm{H}), 2.3(\mathrm{~m}, 1 \mathrm{H}), 2.1-2.2\left(\mathrm{~m}, 8 \mathrm{H}_{\text {n }} 1.9(\mathrm{~m}, 1 \mathrm{H}), 1.6-1.5\right.$ $(\mathrm{m}, 5 \mathrm{H}), 1.4(\mathrm{~s}, 9 \mathrm{H}), 1.3(\mathrm{~m}, 2 \mathrm{H}), 0.9-0.8(\mathrm{~m}, 18 \mathrm{H}) ;{ }^{13} \mathrm{C} \mathrm{NMR}: \delta=$ $172.6,172.1,172.1,172.0-171.9,171.7,168.8,167.6,167.4,157.5$, $157.4,153.4,134.9,133.5,129.1,125.3,124.3,122.3,116.5,116.2$, $118.0,106.4,106.2,79.5,66.9,66.7,57.9,57.4,56.5,52.8,52.0,51.6$ 40.9, 39.1, 32.6, 31.9, 31.0, 29.3, 28.8, 28.3, 25.1, 24.8, 24.2, 22.6, 22.4, 19.0, 18.6, 17.8; $\mathrm{ESI}^{+} \mathrm{MS} \mathrm{m} / \mathrm{z}: 1114[\mathrm{M}+\mathrm{Na}]^{+}$; Anal. $(\mathrm{C}, \mathrm{H}, \mathrm{N})$ : $\mathrm{C}_{56} \mathrm{H}_{82} \mathrm{~N}_{8} \mathrm{O}_{14}$.

Synthesis of molecular tong 6 . TFA ( $2 \mathrm{~mL}, 26 \mathrm{mmol})$ was added to a solution of $5(100 \mathrm{mg}, 0.09 \mathrm{mmol})$ in dry $\mathrm{CH}_{2} \mathrm{Cl}_{2}(2 \mathrm{~mL})$. The mixture stirred for $2 \mathrm{~h}$, then the solvent was evaporated under reduced pressure. Azeotropic removal of excess TFA was carried out using toluene. The crude product was recrystallized from $\mathrm{MeOH} /$ $\mathrm{Et}_{2} \mathrm{O}$ to give product 6 as a light pink solid: $\mathrm{mp}: 136-139^{\circ} \mathrm{C}$ (79 mg, 78\%); ${ }^{1} \mathrm{H}$ NMR ([D 6 ]DMSO): $\delta=10.1$ (bs, $\left.1 \mathrm{H}\right), 9.9$ (bs, $\left.2 \mathrm{H}\right)$, 8.0-7.7 (m, 8H), 7.2-7.0 (m, 3H), $6.9(\mathrm{~s}, 2 \mathrm{H}), 4.4(\mathrm{bs}, 1 \mathrm{H}), 4.1-4.0$ $(\mathrm{m}, 6 \mathrm{H}), 3.8(\mathrm{~m}, 4 \mathrm{H}), 3.6(\mathrm{~s}, 3 \mathrm{H}), 3.0(\mathrm{~m}, 2 \mathrm{H}), 2.5-2.4(\mathrm{~m}, 4 \mathrm{H}), 2.0$ $(\mathrm{m}, 10 \mathrm{H}), 1.78(\mathrm{~m}, 2 \mathrm{H}), 1.6-1.4(\mathrm{~m}, 8 \mathrm{H}), 0.9-0.7(\mathrm{~m}, 18 \mathrm{H}) ;{ }^{13} \mathrm{C} \mathrm{NMR}$ ([D $\left.\left.\mathrm{D}_{6}\right] \mathrm{DMSO}\right): \delta=172.2,171.7,171.4,170.9,168.0,157.0,152.6$, $135.7,132.6,128.9,124.5,123.7,121.3,115.9,112.5,106.1,66.9$, $57.8,57.2,56.2,51.6,51.1,50.8,40.7,32.0,31.8,31.6,30.2,29.8$, 25.0, 24.9, 24.1, 23.8, 22.9, 21.6, 21.4, 19.16, 18.8, 18.2, 18.1; ESI ${ }^{-}$MS $\mathrm{m} / \mathrm{z}: 990[\mathrm{M}-\mathrm{H}]^{+}$; Anal. $(\mathrm{C}, \mathrm{H}, \mathrm{N}):\left(\mathrm{C}_{51} \mathrm{H}_{74} \mathrm{~N}_{8} \mathrm{O}_{12} \cdot \mathrm{CF}_{3} \mathrm{COOH} \cdot 1.5 \mathrm{H}_{2} \mathrm{O}\right.$.

Synthesis of molecular tong 7 . Tong 7 was synthesized from 27, following the same procedure as described for 1 from 25, and was obtained as a white solid in $54 \%$ yield: $\mathrm{mp}$ : $191-196{ }^{\circ} \mathrm{C} ;{ }^{1} \mathrm{H}$ NMR ([D $\left.\left.\mathrm{D}_{6}\right] \mathrm{DMSO}\right): \delta=9.16(\mathrm{~s}, 1 \mathrm{H}), 8.25(\mathrm{~d}, J=8.0 \mathrm{~Hz}, 1 \mathrm{H}), 8.20(\mathrm{~d}, J=$ $7.0 \mathrm{~Hz}, 1 \mathrm{H}), 7.69(\mathrm{~d}, J=8.9 \mathrm{~Hz}, 1 \mathrm{H}), 7.62(\mathrm{~s}, 1 \mathrm{H}), 7.31(\mathrm{~d}, J=7.0 \mathrm{~Hz}$ $1 \mathrm{H}), 7.21(\mathrm{~s}, 1 \mathrm{H}), 7.16(\mathrm{~s}, 1 \mathrm{H}), 6.97(\mathrm{~d}, J=8.9 \mathrm{~Hz}, 1 \mathrm{H}), 6.23(\mathrm{t}, J=$ $7.0 \mathrm{~Hz}, 1 \mathrm{H}), 4.56(\mathrm{~s}, 2 \mathrm{H}), 4.37(\mathrm{t}, J=8 \mathrm{~Hz}, 1 \mathrm{H}), 4.06(\mathrm{t}, J=7 \mathrm{~Hz}, 2 \mathrm{H})$, $2.43(\mathrm{t}, J=6.6 \mathrm{~Hz}, 2 \mathrm{H}), 2.11(\mathrm{~m}, 1 \mathrm{H}), 2.02(\mathrm{~m}, 2 \mathrm{H}), 0.89(\mathrm{~d}, J=$ $6.4 \mathrm{~Hz}, 6 \mathrm{H}) ;{ }^{13} \mathrm{C}$ NMR $\left(100 \mathrm{MHz},\left[\mathrm{D}_{6}\right] \mathrm{DMSO}\right): \delta=172.3,170.7,168.3$, $157.0,156.7,135.7,133.2,128.9,128.0,123.7,122.5,115.9,106.2$, 104.6, 66.9, 58.9, 51.3, 31.6, 29.7, 25.0, 19.2, 18.0; ESI ${ }^{+} \mathrm{MS} m / z: 851$ $[\mathrm{M}+\mathrm{Na}]^{+}, 867[\mathrm{M}+\mathrm{K}]^{+}$; Anal. $(\mathrm{C}, \mathrm{H}, \mathrm{N}): \mathrm{C}_{42} \mathrm{H}_{52} \mathrm{~N}_{8} \mathrm{O}_{10} \cdot 2 \mathrm{H}_{2} \mathrm{O}$.

Synthesis of molecular tong $\mathbf{8}$. Tong $\mathbf{8}$ was synthesized from $\mathbf{2 8}$ following the same procedure as described for 1 from 25, and was obtained as a white solid in $89 \%$ yield: $\mathrm{mp}$ : $224-246{ }^{\circ} \mathrm{C}$; ${ }^{1} \mathrm{H}$ NMR $\left(400 \mathrm{MHz},\left[\mathrm{D}_{6}\right] \mathrm{DMSO}\right): \delta=10.32(\mathrm{~s}, 1 \mathrm{H}), 9.93(\mathrm{~s}, 2 \mathrm{H}), 8.00(\mathrm{~d}, J=$ $9.0 \mathrm{~Hz}, 1 \mathrm{H}), 7.94(\mathrm{~s}, 1 \mathrm{H}), 7.74(\mathrm{dd}, J=2.7,9.0 \mathrm{~Hz}, 1 \mathrm{H}), 7.70(\mathrm{~d}, J=$ $9.0 \mathrm{~Hz}, 1 \mathrm{H}), 7.18(\mathrm{bd}, J=2 \mathrm{~Hz}, 1 \mathrm{H}), 7.09(\mathrm{~d}, J=9.0 \mathrm{~Hz}, 1 \mathrm{H}), 6.97(\mathrm{dd}$ $J=2.0,9.0 \mathrm{~Hz}, 1 \mathrm{H}), 4.33(\mathrm{~m}, 1 \mathrm{H}), 4.05(\mathrm{t}, J=6.5 \mathrm{~Hz}, 2 \mathrm{H}), 3.84(\mathrm{~s}$ $3 \mathrm{H}), 2.39(\mathrm{~m}, 2 \mathrm{H}), 2.01(\mathrm{~s}, 3 \mathrm{H}), 1.98(\mathrm{~m}, 3 \mathrm{H}), 0.95(\mathrm{~d}, J=6.0 \mathrm{~Hz}, 3 \mathrm{H})$, $0.90(\mathrm{~d}, J=6.0 \mathrm{~Hz}, 3 \mathrm{H}) ;{ }^{13} \mathrm{C}$ NMR $\left(100 \mathrm{MHz},\left[\mathrm{D}_{6}\right.\right.$ ]DMSO): $\delta=171.6$, 169.5, 168.0, 163.3, 157.0, 152.6, 135.7, 132.6, 128.9, 123.7, 123.3, $121.3,121.1,115.9,112.4,106.1,66.9,56.1,31.5,30.7,25.0,19.2$, 18.3; IR $\left(\mathrm{cm}^{-1}\right): 3269,1635,1605,1542,1489,1210(\mathrm{C}-\mathrm{O})$; $\mathrm{ESI}^{+} \mathrm{MS}$ $\mathrm{m} / \mathrm{z}: 964[\mathrm{M}+\mathrm{Na}]^{+}, 980[\mathrm{M}+\mathrm{K}]^{+}$; Anal. $(\mathrm{C}, \mathrm{H}, \mathrm{N}): \mathrm{C}_{48} \mathrm{H}_{60} \mathrm{~N}_{8} \mathrm{O}_{12} \cdot 1.5 \mathrm{H}_{2} \mathrm{O}$.

Synthesis of molecular tong 9. Tong 9 was synthesized from 19, following the same procedure as described for 1 from 25 , and was obtained as a white solid in $82 \%$ yield: $\mathrm{mp}$ : $183-185^{\circ} \mathrm{C} ;{ }^{1} \mathrm{H}$ NMR 
(400 MHz, [D $\left.\left.\mathrm{D}_{6}\right] \mathrm{DMSO}\right): \delta=9,19(\mathrm{~s}, 1 \mathrm{H}), 8.36(\mathrm{~d}, J=7.3 \mathrm{~Hz}, 1 \mathrm{H}), 8.19$ $(\mathrm{dd}, J=1.2,7.1 \mathrm{~Hz}, 1 \mathrm{H}), 7.69(\mathrm{~d}, J=8.9 \mathrm{~Hz}, 1 \mathrm{H}), 7.61(\mathrm{~s}, 1 \mathrm{H}), 7.34-$ $7.29(\mathrm{~m}, 6 \mathrm{H}), 7.20(\mathrm{~s}, 1 \mathrm{H}), 7.16(\mathrm{~s}, 1 \mathrm{H}), 6.97(\mathrm{dd}, J=2.2,8.9 \mathrm{~Hz}, 1 \mathrm{H})$, $6.23(\mathrm{t}, J=7.1 \mathrm{~Hz}, 1 \mathrm{H}), 5.00(\mathrm{~s}, 2 \mathrm{H}), 4.56(\mathrm{~s}, 2 \mathrm{H}), 4.40(\mathrm{bm}, 1 \mathrm{H}), 4.06$ $(\mathrm{t}, J=6.3 \mathrm{~Hz}, 2 \mathrm{H}), 2.96(\mathrm{q}, J=6.3 \mathrm{~Hz}, 2 \mathrm{H}), 2.39(\mathrm{t}, J=6.4 \mathrm{~Hz}, 2 \mathrm{H})$, 2.02 (quint, $J=6.4 \mathrm{~Hz}, 2 \mathrm{H}), 1.74(\mathrm{~m}, 1 \mathrm{H}), 1.58(\mathrm{~m}, 1 \mathrm{H}), 1.39(\mathrm{~m}, 2 \mathrm{H})$, $1.32(\mathrm{~m}, 2 \mathrm{H}) ;{ }^{13} \mathrm{C}$ NMR $\left(100 \mathrm{MHz},\left[\mathrm{D}_{6}\right] \mathrm{DMSO}\right): \delta=172.3,171.3,168.3$, $157.0,156.7,156.1,137.3,135.7,133.1,133,128.9,128.3,128.1$, $127.7,123.7,122.3,115.9,106.2,104.7,66.9,65.1,53.7,51.3,40.1$, 31.7, 30.7, 29.1, 24.9, 22.8; IR ( $\left.\mathrm{cm}^{-1}\right): 3303,1676,1647,1515,1210$; $\mathrm{ESI}^{+} \mathrm{MS} \mathrm{m} / \mathrm{z}: 1178[\mathrm{M}+\mathrm{Na}]^{+}$; Anal. $(\mathrm{C}, \mathrm{H}, \mathrm{N}): \mathrm{C}_{60} \mathrm{H}_{70} \mathrm{~N}_{10} \mathrm{O}_{14} \cdot 2 \cdot 5 \mathrm{H}_{2} \mathrm{O}$.

Synthesis of molecular tong 10. $\mathrm{Pd} / \mathrm{C} 10 \%$ (12 mg, $20 \%$ mass) was added to a solution of $\mathbf{9}(65 \mathrm{mg}, 0.06 \mathrm{mmol})$ in $\mathrm{MeOH}(20 \mathrm{~mL})$ and DMF $(2 \mathrm{~mL})$. The reaction flask was purged three times with hydrogen, and stirring was maintained under hydrogen atmosphere at room temperature for $12 \mathrm{~h}$. The mixture was filtered through Celite, and the cake was washed with $\mathrm{MeOH}(200 \mathrm{~mL})$. The filtrate was concentrated under reduced pressure and the resulting residue was dissolved in $\mathrm{MeOH}$. TFA $(0.02 \mathrm{~mL}, 0.26 \mathrm{mmol}$, 4.3 equiv) was added, and the mixture stirred for $10 \mathrm{~min}$ before evaporation of the solvent under reduced pressure. The crude product was recrystallized from $\mathrm{MeOH} / \mathrm{Et}_{2} \mathrm{O}$ to obtain 10 as a beige solid: $\mathrm{mp}: 176-178{ }^{\circ} \mathrm{C}(45 \mathrm{mg}, 72 \%) . R_{\mathrm{f}}=0(\mathrm{EtOAc} / \mathrm{MeOH} /$ $\left.\mathrm{NH}_{4} \mathrm{OH}, 79: 20: 1\right)$; ${ }^{1} \mathrm{H}$ NMR (400 MHz, [D $]$ DMSO): $\delta=9.21(\mathrm{~s}, 1 \mathrm{H})$, $8.39(\mathrm{~d}, J=8.0 \mathrm{~Hz}, 1 \mathrm{H}), 8.27$ (bs, $3 \mathrm{H}), 8.19(\mathrm{~d}, J=8.0 \mathrm{~Hz}, 1 \mathrm{H}), 7.71$ (d, J=8.8, 1H), $7.63(\mathrm{~s}, 1 \mathrm{H}), 7.32(\mathrm{~d}, J=8.0 \mathrm{~Hz}, 1 \mathrm{H}), 7.20(\mathrm{~s}, 1 \mathrm{H})$, $7.18(\mathrm{~s}, 1 \mathrm{H}), 6.97(\mathrm{~d}, J=8.8 \mathrm{~Hz}, 1 \mathrm{H}), 6.24(\mathrm{t}, J=8.0 \mathrm{~Hz}, 1 \mathrm{H}), 4.57(\mathrm{~s}$, $2 \mathrm{H}), 4.46(\mathrm{bm}, 1 \mathrm{H}), 4.06(\mathrm{~m}, 2 \mathrm{H}), 2.97(\mathrm{~m}, 2 \mathrm{H}), 2.40(\mathrm{t}, J=7.2 \mathrm{~Hz}$, $2 \mathrm{H}), 2.03(\mathrm{~m}, 2 \mathrm{H}), 1.77(\mathrm{~m}, 1 \mathrm{H}), 1.60(\mathrm{~m}, 1 \mathrm{H}), 1.40-1.33(\mathrm{~m}, 4 \mathrm{H})$; ${ }^{13} \mathrm{C}$ NMR $\left(100 \mathrm{MHz},\left[\mathrm{D}_{6}\right] \mathrm{DMSO}\right): \delta=172.0,170.1,168.3,156.2,135.7$, 133.6, 129.0, 128.7, 123.7, 122.6, 115.7, 105.9, 104.8, 66.7, 47.7, 51.0, 31.6, 30.7, 32.0, 24.8, 22.6; IR (cm $\left.{ }^{-1}\right): 3338,1644,1514,1200,1130$; $\mathrm{ESI}^{+} \mathrm{MS} \mathrm{m} / \mathrm{z}: 888[\mathrm{M+H}]^{+}, 910[\mathrm{M}+\mathrm{Na}]^{+}$; Anal. $(\mathrm{C}, \mathrm{H}, \mathrm{N})$ : $\mathrm{C}_{44} \mathrm{H}_{48} \mathrm{~N}_{10} \mathrm{O}_{10} \cdot 2 \mathrm{CF}_{3} \mathrm{COOH} \cdot 1.5 \mathrm{H}_{2} \mathrm{O}$.

Synthesis of molecular tong 11. Tong 11 was synthesized from 23, following the same procedure as described for 1 from 25, and was obtained as a white solid in $74 \%$ yield: $\mathrm{mp}: 188-190^{\circ} \mathrm{C}$; ${ }^{1} \mathrm{H}$ NMR $\left(400 \mathrm{MHz}\right.$, [D $\mathrm{D}_{6}$ DMSO): $\delta=10.33(\mathrm{~s}, 1 \mathrm{H}), 9.93(\mathrm{~m}, 2 \mathrm{H}), 8.06$ (d, $J=8.1 \mathrm{~Hz}, 1 \mathrm{H}), 7.94(\mathrm{~d}, J=2.6,1 \mathrm{H}), 7.76(\mathrm{dd}, J=2.6,9.0 \mathrm{~Hz}, 1 \mathrm{H})$, $7.69(\mathrm{~d}, J=9.0 \mathrm{~Hz}, 1 \mathrm{H}), 7.35-7.31(\mathrm{~m}, 5 \mathrm{H}), 7.21(\mathrm{bs}, 1 \mathrm{H}), 7.18(\mathrm{~s}$, $1 \mathrm{H}), 7.09(\mathrm{~d}, J=9.0 \mathrm{~Hz}, 1 \mathrm{H}), 6.96(\mathrm{dd}, J=2.0,9.0 \mathrm{~Hz}, 1 \mathrm{H}), 5.00(\mathrm{~s}$, $2 \mathrm{H}), 4.41(\mathrm{bm}, 1 \mathrm{H}), 4.04(\mathrm{t}, J=6.1,2 \mathrm{H}), 3.84(\mathrm{~s}, 3 \mathrm{H}), 2.98(\mathrm{q}, J=$ $6.1 \mathrm{~Hz}, 2 \mathrm{H}), 2.36(\mathrm{t}, J=7.2 \mathrm{~Hz}, 2 \mathrm{H}), 2.01(\mathrm{~s}, 3 \mathrm{H}), 1.98(\mathrm{~m}, 2 \mathrm{H}), 1.69$ $(\mathrm{bm}, 1 \mathrm{H}), 1.58(\mathrm{bm}, 1 \mathrm{H}), 1.40(\mathrm{~m}, 2 \mathrm{H}), 1.34(\mathrm{~m}, 2 \mathrm{H}) ;{ }^{13} \mathrm{C} \mathrm{NMR}$ (100 MHz, $\left[\mathrm{D}_{6}\right.$ ]DMSO): $\delta=171.6,170.2,168.0,163.1,157.0,156.1$, 152.7, 137.3, 135.8, 132.6, 128.9, 128.3, 127.7, 123.7, 123.4, 121.3, $121.0,115.9,112.4,106.2,66.9,65.1,56.1,50.9,40.1,32.0,31.6,29.1$, 24.9, 23.8, 22.6; IR $\left(\mathrm{cm}^{-1}\right): 3261,1686,1605,1539,1252,1210$, 1023; $\mathrm{ESI}^{+} \mathrm{MS} \mathrm{m} / \mathrm{z}: 1290[\mathrm{M}+\mathrm{Na}]^{+}, 1306[\mathrm{M+K}]^{+}$; Anal. $(\mathrm{C}, \mathrm{H}, \mathrm{N})$ : $\mathrm{C}_{66} \mathrm{H}_{78} \mathrm{~N}_{10} \mathrm{O}_{16} \cdot 1.5 \mathrm{H}_{2} \mathrm{O}$.

Synthesis of molecular tong $\mathbf{1 2}$. Tong 12 was synthesized from 11, following the same procedure as described for 10 from 9 , and was obtained as a white solid in $69 \%$ yield: $\mathrm{mp}: 139-141^{\circ} \mathrm{C}$; ${ }^{1} \mathrm{H}$ NMR $(400 \mathrm{MHz}$, [D 6 ]DMSO): $\delta=10.35(\mathrm{~s}, 1 \mathrm{H}), 9.97(\mathrm{~d}, J=8.9 \mathrm{~Hz}$, $2 \mathrm{H}), 8.51(\mathrm{bs}, 3 \mathrm{H}), 8.13(\mathrm{~d}, J=7.3 \mathrm{~Hz}, 1 \mathrm{H}), 7.98(\mathrm{~s}, 1 \mathrm{H}), 7.70(\mathrm{~d}, J=$ $8.4 \mathrm{~Hz}, 2 \mathrm{H}), 7.19(\mathrm{~s}, 1 \mathrm{H}), 7.10(\mathrm{~d}, J=8.8 \mathrm{~Hz}, 1 \mathrm{H}), 6.98(\mathrm{~d}, J=7.9 \mathrm{~Hz}$, $1 \mathrm{H}), 4.43(\mathrm{~m}, 1 \mathrm{H}), 4.06(\mathrm{~m}, 2 \mathrm{H}), 3.83(\mathrm{~s}, 3 \mathrm{H}), 2.84(\mathrm{~m}, 2 \mathrm{H}), 2.36(\mathrm{~m}$, $2 \mathrm{H}), 2.01(\mathrm{~s}, 3 \mathrm{H}), 1.72(\mathrm{~m}, 2 \mathrm{H}), 1.57(\mathrm{~m}, 1 \mathrm{H}), 1.38(\mathrm{~m}, 2 \mathrm{H}), 1.29-$ $1.23(\mathrm{~m}, 2 \mathrm{H}) ;{ }^{13} \mathrm{C}$ NMR $\left(100 \mathrm{MHz},\left[\mathrm{D}_{6}\right.\right.$ ]DMSO): $\delta=171.7,170.2,168.0$, $163.3,158.5-157.6(q, J=32 \mathrm{~Hz}), 157.0,152.7,135.8,132.6,129.0$, 123.7, 123.4, 121.3, 121.0, 118.8, 115.9, 112.4, 106.2, 66.9, 56.1, 50.7, 48.0, 31.7, 31.6, 29.0, 24.9, 23.8, 22.2; IR $\left(\mathrm{cm}^{-1}\right): 1648,1513,1128$;
$\mathrm{ESI}^{+} \quad \mathrm{MS} \quad \mathrm{m} / \mathrm{z}: 1000 \quad[\mathrm{M+H}]^{+}$; $\quad$ Anal. $(\mathrm{C}, \mathrm{H}, \quad \mathrm{N})$ : $\mathrm{C}_{50} \mathrm{H}_{66} \mathrm{~N}_{10} \mathrm{O}_{12} \cdot 2 \mathrm{CF}_{3} \mathrm{COOH} \cdot 3.5 \mathrm{H}_{2} \mathrm{O}$.

Synthesis of molecular tong 13. Tong 13 was synthesized from 20, following the same procedure as described for 1 from 25 , and was obtained as a white solid in $69 \%$ yield: $\mathrm{mp}$ : $149-151^{\circ} \mathrm{C}$; ${ }^{1} \mathrm{H}$ NMR $\left(400 \mathrm{MHz},\left[\mathrm{D}_{6}\right] \mathrm{DMSO}\right): \delta=9.15(\mathrm{~s}, 1 \mathrm{H}), 8.21(\mathrm{~d}, J=7.0 \mathrm{~Hz}$, $1 \mathrm{H}), 7.83(\mathrm{t}, J=5.2,1 \mathrm{H}), 7.70(\mathrm{~d}, J=8.8 \mathrm{~Hz}, 1 \mathrm{H}), 7.61(\mathrm{~s}, 1 \mathrm{H}), 7.37$ $(\mathrm{d}, J=7.0 \mathrm{~Hz}, 1 \mathrm{H}), 7.30(\mathrm{~d}, J=7.0 \mathrm{~Hz}, 1 \mathrm{H}), 7.20(\mathrm{~s}, 1 \mathrm{H}), 7.18(\mathrm{~s}, 1 \mathrm{H})$, $6.95(\mathrm{dd}, J=1.6,8.8 \mathrm{~Hz}, 1 \mathrm{H}), 6.23(\mathrm{t}, J=7.0 \mathrm{~Hz}, 1 \mathrm{H}), 4.57(\mathrm{~s}, 2 \mathrm{H})$, $4.04(\mathrm{~m}, 3 \mathrm{H}), 3.03(\mathrm{bq}, J=5.8 \mathrm{~Hz}, 2 \mathrm{H}), 2.25(\mathrm{t}, J=7.0,2 \mathrm{H}), 1.98(\mathrm{q}$, $J=7.0 \mathrm{~Hz}, 2 \mathrm{H}), 1.71(\mathrm{~m}, 1 \mathrm{H}), 1.55(\mathrm{~m}, 1 \mathrm{H}), 1.38(\mathrm{~s}, 9 \mathrm{H}), 1.38-1.31$ $(\mathrm{m}, 4 \mathrm{H}) ;{ }^{13} \mathrm{C}$ NMR $\left(100 \mathrm{MHz},\left[\mathrm{D}_{6}\right] \mathrm{DMSO}\right): \delta=171.8,171.4,168.3$, 157.0, 156.7, 155.7, 135.8, 133.0, 129.0, 128.0, 123.7, 122.1, 115.9, 106.1, 104.7, 78.5, 67.0, 55.4, 51.2, 38.3, 31.8, 30.8, 28.8, 28.2, 24.9, 23.1; IR $\left(\mathrm{cm}^{-1}\right): 3314,2933,1684,1645,1514,1369,1252,1210$, 1163; $\mathrm{ESI}^{-}$MS m/z: $1086[M-1]^{+}$. Anal (C, H, N): $\mathrm{C}_{54} \mathrm{H}_{76} \mathrm{~N}_{10} \mathrm{O}_{14} \cdot 1.5 \mathrm{H}_{2} \mathrm{O}$.

Synthesis of molecular tong 14. Tong 14 was synthesized from 13 , following the same procedure as described for 4 , and was obtained as a white solid in $99 \%$ yield: $\mathrm{mp}: 148-150{ }^{\circ} \mathrm{C}$; ${ }^{1} \mathrm{H}$ NMR $\left(400 \mathrm{MHz},\left[\mathrm{D}_{6}\right] \mathrm{DMSO}\right): \delta=9.98(\mathrm{~s}, 1 \mathrm{H}), 8.21(\mathrm{~d}, J=1.5 \mathrm{~Hz}, 1 \mathrm{H}), 8.17$ $(\mathrm{s}, 3 \mathrm{H}), 7.82(\mathrm{t}, J=5.3 \mathrm{~Hz}, 1 \mathrm{H}), 7.68(\mathrm{~d}, J=9.0 \mathrm{~Hz}, 1 \mathrm{H}), 7.64(\mathrm{~s}, 1 \mathrm{H})$, 7.37 (dd, $J=1.5,7.0 \mathrm{~Hz}, 1 \mathrm{H}), 7.20(\mathrm{~s}, 1 \mathrm{H}), 7.16(\mathrm{~d}, J=2.0,1 \mathrm{H}), 6.94$ $(\mathrm{dd}, J=2.2,9.0 \mathrm{~Hz}, 1 \mathrm{H}), 6.25(\mathrm{t}, J=7.0 \mathrm{~Hz}, 1 \mathrm{H}), 4.57(\mathrm{~s}, 2 \mathrm{H}), 4.24(\mathrm{t}$, $J=6.0 \mathrm{~Hz}, 1 \mathrm{H}), 4.02(\mathrm{t}, J=6.0 \mathrm{~Hz}, 2 \mathrm{H}), 3.02(\mathrm{q}, J=7.0 \mathrm{~Hz}, 2 \mathrm{H}), 2.23$ $(\mathrm{t}, J=7.0 \mathrm{~Hz}, 2 \mathrm{H}), 1.95$ (quint, $J=7.0 \mathrm{~Hz}, 2 \mathrm{H}), 1.73(\mathrm{~m}, 2 \mathrm{H}), 1.39(\mathrm{q}$, $J=7.0 \mathrm{~Hz}, 2 \mathrm{H}), 1.33(\mathrm{~m}, 2 \mathrm{H}) ;{ }^{13} \mathrm{C}$ NMR $\left(100 \mathrm{MHz},\left[\mathrm{D}_{6}\right] \mathrm{DMSO}\right): \delta=$ $171.4,168.5,168.3,157.0,156.7,135.7,134.2,129,127.7,125.0$, 123.6, 116.0, 106.1, 104.4, 67.0, 52.6, 51.2, 38.2, 31.8, 31.1, 28.7, 24.9, 21.7; IR $\left(\mathrm{cm}^{-1}\right): 3262,2968,1633,1514,1200,1130 ; \mathrm{ESI}^{+} \mathrm{MS}$ $\mathrm{m} / \mathrm{z}: \quad 888[\mathrm{M+H}]^{+}, \quad 910[\mathrm{M}+\mathrm{Na}]^{+}$; Anal. $(\mathrm{C}, \mathrm{H}, \quad \mathrm{N})$ : $\mathrm{C}_{44} \mathrm{H}_{58} \mathrm{~N}_{10} \mathrm{O}_{10} \cdot 2 \mathrm{CF}_{3} \mathrm{COOH} \cdot 2 \mathrm{H}_{2} \mathrm{O}$.

Synthesis of molecular tong 15 . Tong 15 was synthesized from 24, following the same procedure as described for 1 from 25, and was obtained as a white solid in $67 \%$ yield: $\mathrm{mp}$ : $164-170^{\circ} \mathrm{C}$; ${ }^{1} \mathrm{H}$ NMR $\left(400 \mathrm{MHz}, \mathrm{CDCl}_{3}\right): \delta=11.85$ (bs, $\left.1 \mathrm{H}\right), 11.25$ (bs, $\left.1 \mathrm{H}\right), 8.92$ (s, $1 \mathrm{H}), 8.40(\mathrm{bm}, 1 \mathrm{H}), 7.95(\mathrm{~s}, 1 \mathrm{H}), 7.54(\mathrm{~d}, J=8.6 \mathrm{~Hz}, 1 \mathrm{H}), 6.97(\mathrm{~d}, J=$ $8.9 \mathrm{~Hz}, 1 \mathrm{H}), 6.89(\mathrm{~s}, 1 \mathrm{H}), 6.86(\mathrm{~d}, J=8.6 \mathrm{~Hz}, 1 \mathrm{H}), 5.86(\mathrm{bs}, 1 \mathrm{H}), 5.66$ $(\mathrm{d}, J=7.8 \mathrm{~Hz}, 1 \mathrm{H}), 4.88(\mathrm{bm}, 1 \mathrm{H}), 4.00(\mathrm{~m}, 5 \mathrm{H}), 3.08(\mathrm{~m}, 2 \mathrm{H}), 2.27$ $(\mathrm{m}, 2 \mathrm{H}), 2.19(\mathrm{~s}, 3 \mathrm{H}), 2.09(\mathrm{~m}, 2 \mathrm{H}), 1.80(\mathrm{~m}, 1 \mathrm{H}), 1.71(\mathrm{~m}, 1 \mathrm{H}), 1.45$ $(\mathrm{s}, 9 \mathrm{H}), 1.34-1.32(\mathrm{~m}, 4 \mathrm{H}) ;{ }^{13} \mathrm{C}$ NMR $\left(100 \mathrm{MHz},\left[\mathrm{D}_{6}\right] \mathrm{DMSO}\right): \delta=172.4$, 171.0, 169.2, 167.0, 157.3, 155.6, 153.6, 135.8, 133.1, 129.0, 126.2, $124.2,122.6,117.9,116.1,112.1,106.3,79.8,66.7,56.6,52.1,39.3$, $34.0,32.7,29.2,28.4,25.1,24.5,22.1$; IR $\left(\mathrm{cm}^{-1}\right): 3286,2936,1631$, 1493, 1251, 1161; $\mathrm{ESI}^{+} \mathrm{MS} \mathrm{m} / \mathrm{z}: 1222[\mathrm{M}+\mathrm{Na}]^{+}$; Anal. $(\mathrm{C}, \mathrm{H}, \mathrm{N})$ : $\mathrm{C}_{60} \mathrm{H}_{82} \mathrm{~N}_{10} \mathrm{O}_{16} \cdot 4 \mathrm{H}_{2} \mathrm{O}$.

Synthesis of molecular tong 16 . Tong 16 was synthesized from 15 , following the same procedure as described for 4 , and was obtained as a white solid in $92 \%$ yield: $\mathrm{mp}$ : $169-171{ }^{\circ} \mathrm{C}$; ${ }^{1} \mathrm{H}$ NMR $\left(400 \mathrm{MHz},\left[\mathrm{D}_{6}\right] \mathrm{DMSO}\right): \delta=10.13$ (bs, $\left.1 \mathrm{H}\right), 9.98$ (bs, $\left.1 \mathrm{H}\right), 8.69$ (bs, $1 \mathrm{H}), 7.97(\mathrm{~d}, J=2.5 \mathrm{~Hz}, 1 \mathrm{H}), 7.86(\mathrm{t}, J=5.1 \mathrm{~Hz}, 1 \mathrm{H}), 7.72(\mathrm{dd}, J=2.5$, $9.0 \mathrm{~Hz}, 1 \mathrm{H}), 7.70(\mathrm{~d}, J=8.7 \mathrm{~Hz}, 1 \mathrm{H}), 7.16(\mathrm{~s}, 1 \mathrm{H}), 7.12(\mathrm{~d}, J=9.0 \mathrm{~Hz}$, $1 \mathrm{H}), 6.94(\mathrm{dd}, J=2.1,8.7 \mathrm{~Hz}, 1 \mathrm{H}), 4.04(\mathrm{t}, J=6.2 \mathrm{~Hz}, 2 \mathrm{H}), 3.85(\mathrm{~s}$, $3 \mathrm{H}), 3.83(\mathrm{~m}, 1 \mathrm{H}), 3.06(\mathrm{~m}, 2 \mathrm{H}), 2.28(\mathrm{t}, J=7.0 \mathrm{~Hz}, 2 \mathrm{H}), 2.01(\mathrm{~s}, 3 \mathrm{H})$, $1.99(\mathrm{~m}, 2 \mathrm{H}), 1.78(\mathrm{bm}, 2 \mathrm{H}), 1.43(\mathrm{~m}, 4 \mathrm{H}) ;{ }^{13} \mathrm{C}$ NMR $(100 \mathrm{MHz}$, [D $\mathrm{D}_{6}$ ]DMSO): $\delta=171.5,168.1,167.2,163.5,157.0,152.6,135.8,132.7$, 129.0, 123.7, 123.5, 121.2, 121.0, 115.9, 112.5, 106.1, 67.0, 56.2, 51.2, $38.2,31.9,31.0,28.7,25.0,23.8,21.5$; IR $\left(\mathrm{cm}^{-1}\right): 3316,2948,1632$, 1490, 1252, 1133, 1016; ESI ${ }^{+}$MS m/z: $1000[M+1]^{+}$; Anal. $(C, H, N)$ : $\mathrm{C}_{50} \mathrm{H}_{66} \mathrm{~N}_{10} \mathrm{O}_{12} \cdot 2 \mathrm{CF}_{3} \mathrm{COOH} \cdot 2 \mathrm{H}_{2} \mathrm{O}$. 


\section{Enzymatic studies}

The fluorogenic substrate DABCYL- $\gamma$-abu-Ser-GIn-Asn-Tyr-Pro-lleVal-GIn-EDANS \{DABCYL: 4-(4'-dimethylaminophenylazo)benzoyl; $\gamma$ abu: $\gamma$-amino butyric acid; EDANS: 5-[(2-aminoethyl)amino]naphthalene-1-sulfonic acid)\} was purchased from Bachem (Germany). Other reagents and solvents were purchased from various commercial sources as well. Absorbance measurements were obtained using a spectrofluorimeter PerkinElmer LS50B. Fluorescence intensities were measured using a BMG Fluostar microplate reader.

\section{Wild-type and mutated proteases}

The HIV-1 protease enzymes (WT and ANAM-11 $1^{[21]}$ ) used in this study were expressed and purified as previously described. ${ }^{[14]}$ They were produced in E. coli using the pET-9 expression vector and Rosetta(DE3)pLysS host bacterium cell strain. The protease domain of wild-type PR includes five protective mutations: Q7K, L33I, and L63I to minimize autoproteolysis, and C67A and C95A to prevent cysteine-thiol oxidation. ANAM-11 contains the mutations L10I, M36I, S37D, M46I, R57K, L63P, A71V, G73S, I84V, L90M, and 193L. ${ }^{[21]}$

\section{Enzyme and inhibition assays}

The proteolytic activities of wild-type and mutated proteases were determined fluorometrically using the fluorogenic substrate DABCYL- $\gamma$-abu-SQNYPIVQ-EDANS $\left(\lambda_{\mathrm{ex}}=340 \mathrm{~nm} ; \lambda_{\mathrm{em}}=490 \mathrm{~nm}\right)$ in $100 \mathrm{~mm}$ sodium acetate, $1 \mathrm{~mm}$ EDTA, and $1 \mathrm{M} \mathrm{NaCl}$ at $\mathrm{pH} 4.7$ and $30^{\circ} \mathrm{C}$ (final volume $=150 \mu \mathrm{L}$ ). The substrate and test compound were first dissolved in DMSO, with a final DMSO concentration of $3 \%(v / v)$. The mechanism of inhibition and corresponding kinetic constants $K_{\text {id }}$ (dimerization inhibition) or $K_{\text {ic }}$ (competitive inhibition) were determined using Zhang-Poorman kinetic analysis. ${ }^{[10]}$ Kinetic experiments were carried out at constant substrate concentration $(5.2 \mu \mathrm{M})$ with at least six enzyme concentrations (5.33-18.6 nM) and a range of inhibitor concentrations $(1-28 \mu \mathrm{M})$. Experimental data were fitted according to reference 14. All experiments were performed, at minimum, in triplicate.

\section{Acknowledgements}

We thank Claire Troufflard for NMR experiments, the European Community for financial support for A.V. (Marie Curie Early Stage Training Fellowship of the European Community's Sixth Framework Programme, contract MESTCT-2004-515968), the Ministère de la Recherche et des Technologies (MRT), and the Agence Nationale sur la Recherche contre le Sida (ANRS) for financial support for L.D. and L.B., respectively. We also thank Prof. Ernesto Freire of the Department of Biology and Biocalorimetry Center, Johns Hopkins University, Baltimore, MD (USA), for the generous gift of the plasmid encoding the ANAM-11 mutant.
Keywords: dimerization · HIV-1 · inhibitors · peptidomimetics · proteases

[1] S. Oroszlan, R. B. Luftig, Curr. Top. Microbiol. Immunol. 1990, 157, 153 185.

[2] A. Gustchina, I. T. Weber, Proteins 1991, 10, 325-339.

[3] R. Ishima, R. Ghirlando, J. Tözser, A. M. Gronenborn, D. A. Torchia, J. M. Louis, J. Biol. Chem. 2001, 276, 49110-49116.

[4] M. J. Todd, M. Semo, E. Freire, J. Mol. Biol. 1998, 283, 475-488.

[5] A. Carr, Nat. Rev. Drug Discovery 2003, 2, 624-634.

[6] a) R. T. D'Aquila, J. M. Schapiro, F. Brun-Vézinet, B. Clotet, B. Conway, L. M. Demeter, R. M. Grant, V. A. Johnson, D. R. Kuritzkes, C. Loveday, R. W. Shafer, D. D. Richman, Top. HIV Med. 2002, 10, $21-25$; b) E. De Clercq, Nat. Rev. Microbiol. 2004, 2, 704-720.

[7] Y. Koh, S. Matsumi, D. Das, M. Amano, D. A. Davis, J. Li, S. Leschenko, A. Baldridge, T. Shioda, R. Yarchoan, A. K. Ghosh, H. Mitsuya, J. Biol. Chem. 2007, 282, 28709-28720.

[8] a) N. Boggetto, M. Reboud-Ravaux, Biol. Chem. 2002, 383, 1321-1324; b) L. Bannwarth, M. Reboud-Ravaux, Biochem. Soc. Trans. 2007, 35, 551 554.

[9] H. J. Schramm, H. Nakashima, W. Schramm, H. Wakayama, N. Yamamoto, Biochem. Biophys. Res. Commun. 1991, 179, 847-851.

[10] Z. Y. Zhang, R. A. Poorman, L. L. Maggiora, R. L. Heinrikson, F. J. Kézdy, J. Biol. Chem. 1991, 266, $15591-15594$.

[11] J. Franciskovich, K. Houseman, R. Mueller, J. Chiemlevski, Bioorg. Med. Chem. Lett. 1993, 3, 765-768.

[12] H. J. Schramm, E. de Rosny, M. Reboud-Ravaux, J. Buttner, A. Dick, W. Schramm, Biol. Chem. 1999, 380, 593-596.

[13] J. Dumond, N. Boggetto, H. J. Schramm, W. Schramm, M. Takahashi, M. Reboud-Ravaux, Biochem. Pharmacol. 2003, 65, 1097-1102.

[14] L. Bannwarth, T. Rose, L. Dufau, R. Vanderesse, J. Dumond, B. JamartGrégoire, C. Pannecouque, E. De Clercq, M. Reboud-Ravaux, Biochemistry 2009, 48, 379-387.

[15] P. Breccia, N. Boggetto, R. Perez-Fernadez, M. Van Gool, M. Takahashi, L. Rene, P. Prados, B. Badet, M. Reboud-Ravaux, J. de Mendoza, J. Med. Chem. 2003, 46, 5196-5207.

[16] R. Zutshi, J. Franciskovich, M. Slultz, B. Schwetizer, P. Bishop, M. Wilson, J. Chmielewski, J. Am. Chem. Soc. 1997, 119, $4841-4845$.

[17] L. G. Ulysse, J. Chiemlevski, Bioorg. Med. Chem. Lett. 1998, 8, 3281 3286.

[18] A. Bouras, N. Boggetto, Z. Benatalah, E. de Rosny, S. Sicsic, M. ReboudRavaux, J. Med. Chem. 1999, 42, 957-962.

[19] N. Merabet, J. Dumond, B. Collinet, L. Van Baelinghem, N. Boggetto, S. Ongeri, F. Ressad, M. Reboud-Ravaux, S. Sicsic, J. Med. Chem. 2004, 47, $6392-6400$.

[20] L. Bannwarth, A. Kessler, S. Pethe, B. Collinet, M. Merabet, N. Boggetto, S. Sicsic, M. Reboud-Ravaux, S. Ongeri, J. Med. Chem. 2006, 49, 46574664.

[21] S. Muzammil, P. Ross, E. Freire, Biochemistry 2003, 42, 631-638.

Received: July 23, 2010

Revised: September 9, 2010

Published online on October 8, 2010 